PHYSICAL REVIEW D 95, 044044 (2017)

\title{
Stability of singularity-free cosmological solutions in Hořava-Lifshitz gravity
}

\author{
Yosuke Misonoh, ${ }^{*}$ Mitsuhiro Fukushima, ${ }^{\dagger}$ and Shoichiro Miyashita \\ Department of Physics, Waseda University, Okubo 3-4-1, Shinjuku, Tokyo 169-8555, Japan
}

(Received 8 January 2017; published 28 February 2017)

\begin{abstract}
We study the stability of singularity-free cosmological solutions with a positive cosmological constant based on the projectable Hořava-Lifshitz (HL) theory. In the HL theory, the isotropic and homogeneous cosmological solutions with bounce can be realized if the spatial curvature is nonzero. By performing a perturbation analysis around nonflat Friedmann-Lemaitre-Robertson-Walker (FLRW) spacetime, we derive a quadratic action and discuss the stability, i.e., ghost and tachyon-free conditions. Although the squared effective mass of scalar perturbation must be negative in the infrared regime, we can avoid tachyon instability by considering strong Hubble friction. Additionally, we estimate the backreaction from the perturbations on the background geometry, especially against an anisotropic perturbation in closed FLRW spacetime. It turns out that certain types of bouncing solution may be spoiled even if all perturbation modes are stable.
\end{abstract}

DOI: $10.1103 /$ PhysRevD.95.044044

\section{INTRODUCTION}

Spacetime singularity at the beginning of the Universe is a problem of great importance in standard cosmology. According to the singularity theorem proved by Hawking and Penrose [1], a spacetime singularity must have appeared at a finite past. Although the inflationary scenario cures the initial condition problems of the standard cosmological model, the initial singularity cannot be avoided [2]. Since the appearance of a singularity means the breakdown of the classical gravitational theory, one may expect that a fundamental theory beyond general relativity (GR), i.e., the quantum theory of gravity, resolves the problem of infiniteness. A lot of attempts to resolve the singularity at the beginning of the Universe have been proposed based on an extension of GR [3], e.g., the superstring theory [4], loop quantum gravity [5], causal dynamical triangulation [6], and gravity with a nonlocal operator [7]. Regardless of those efforts, the dynamics of the very early Universe is still unclear, because we have not achieved a complete theory of quantum gravity, yet.

Recently, a gravitational theory attracted attention as a candidate for quantum gravity, which is called the HořavaLifshitz (HL) theory [8]. The theory is characterized by Lifshitz scaling [9], which is an anisotropic scaling of spacetime: $t \rightarrow b^{-1} t$ and $\vec{x} \rightarrow b^{-z} \vec{x}$ with dynamical exponent $z$. If we set $z=3$, all types of ultraviolet divergences via Feynman diagrams can be suppressed in fourdimensional spacetime [10]. It means that gravitational interaction can be renormalized by adding appropriate counterterms. Thus, the renormalizable gravitational action

\footnotetext{
*misonoh@aoni.waseda.jp

dark-matter@gravity.phys.waseda.ac.jp

miyashita@gravity.phys.waseda.ac.jp
}

is composed of second-order time derivatives and up to sixth-order spatial derivatives.

Based on the HL theory, ultraviolet spacetime structures have been discussed such as black hole solutions with a universal horizon which is a causal boundary for superluminal propagating modes [11]. In particular, singularity avoidance is an intriguing subject for study. We expect that the quantum gravitational theory resolves spacetime singularities in the classical theory of gravity. The key point to avoid a spacetime singularity is violating the null energy condition. As indicated in Ref. [12], in FriedmannLemaître-Robertson-Walker (FLRW) spacetime with nonzero spatial curvature, higher spatial curvature terms in action avoid evolving into a singularity. Namely, $z=2$ and $z=3$ terms mimic "dark radiation" and "dark stiff matter," respectively. Since the energy densities of these effective matter components depend on coupling constants in the theory, the null energy condition can be violated if the values of the coupling constants are arbitrary. As a result, we can find the bouncing universe and the oscillating universe as singularity-free solutions [13-15].

Although the cosmological singularity avoidance can be realized via higher spatial curvatures as an ultraviolet modification of gravity, one may consider that such solutions show unstable behavior. More specifically, it may possible that the effective matter components derived from $z>1$ Lifshitz scaling terms make spacetime unstable because of a violation of the energy condition. It is reasonable that the spacetime around the Planck scale is perturbed by a quantum fluctuation of gravity. Thus, to examine the spacetime stabilities of these singularity-free solutions is indispensable in order to construct a cosmological scenario without an initial singularity.

In Ref. [15], the dynamics of Bianchi type-IX spacetime, i.e., a spatially homogeneous closed cosmological model, 
is discussed. In other words, the effect of spacetime anisotropy to singularity-free solutions in closed FLRW spacetime is examined. From the result, it is found that the stability against a small anisotropic perturbation depends on the coupling constants of the theory. Thus, we expect that the dynamics of the other types of perturbations are also affected by the coupling constants. Since the HL theory is renormalizable, the values of coupling constants at the Planck scale can be evaluated via beta functions from the renormalization group, in principle. If we obtain the values of coupling constants in the ultraviolet regime, we may predict the dynamics of our Universe at a very early stage, which cannot be directly observed. Thus, we set the goal of this paper to show the stability conditions for singularity-free cosmological solutions against a linearorder perturbation in not only anisotropic modes but also inhomogeneity ones.

The perturbation analysis in FLRW spacetime in the context of the HL theory has been discussed. Without spatial curvature, there is quite a lot of study, e.g., primordial perturbation [16], stability of scalar perturbation $[17,18]$, and stability of de Sitter spacetime [19]. Turning our attention to the case including nonzero spatial curvature, the analyses of scalar perturbation have been performed. In Ref. [20], the authors show a scalar perturbation in vacuum FLRW spacetime, and in Ref. [21], the dynamics of a scalar field in a bouncing universe is discussed. Recalling our motivation to investigate spacetime stabilities of singularity-free solutions, it is necessary to see the dynamics of tensor and vector degree of freedoms as well as scalar ones.

Thus, in this paper, we perform a perturbation analysis regarding tensor, vector, and scalar modes based on the projectable HL theory. Although the nonprojectable HL theory, which is an infrared completion of the projectable HL theory, has been proposed [18], its renormalizablity is still unclear. Therefore, we focus only on the projectable case because of the renormalizable characteristic.

The rest of this paper is organized as follows: In Sec. II, we briefly review the projectable HL theory, especially in the FLRW background. The perturbation theory around FLRW spacetime in the HL theory is discussed in Sec. III. In Sec. IV, we discuss stabilities of the bouncing solution of the HL theory in nonflat FLRW spacetime by investigating ghost and tachyon-free conditions. Additionally, we estimate the backreaction from the perturbation on the background geometry, especially against an anisotropic perturbation in closed FLRW spacetime. Section V is devoted to the conclusion of this paper.

\section{HOŘAVA-LIFSHITZ THEORY IN FLRW SPACETIME}

We briefly review the projectable HL theory, especially in FLRW spacetime. The gravitational action is given by [22]

$$
S=\frac{m_{\mathrm{LV}}^{2}}{2} \int d t d^{3} x\left(\mathcal{L}_{K}+\mathcal{L}_{P}\right),
$$

with

$$
\begin{gathered}
\mathcal{L}_{K}:=N \sqrt{g}\left(\mathcal{K}_{i j} \mathcal{K}^{i j}-\lambda \mathcal{K}^{2}\right), \\
\mathcal{L}_{P}:=-N \sqrt{g}\left[\mathcal{V}_{z=1}+m_{\mathrm{LV}}^{-2} \mathcal{V}_{z=2}+m_{\mathrm{LV}}^{-4} \mathcal{V}_{z=3}\right],
\end{gathered}
$$

where $m_{\mathrm{LV}}$ is the Lorentz-violating mass scale which may be expected around the Planck scale. ${ }^{1}$ The extrinsic curvature $\mathcal{K}_{i j}$ is defined in terms of the lapse function $N$, the shift vector $N_{i}$, and the three-dimensional induced metric $g_{i j}$ :

$$
\mathcal{K}_{i j}:=\frac{1}{2 N}\left[\partial_{t} g_{i j}-\nabla_{i} N_{j}-\nabla_{j} N_{i}\right]
$$

where $\nabla_{i}$ represents the three-dimensional covariant derivative. The potential terms are defined by

$$
\begin{aligned}
\mathcal{V}_{z=1}:= & 2 \Lambda+g_{1} \mathcal{R}, \\
\mathcal{V}_{z=2}:= & g_{2} \mathcal{R}^{2}+g_{3} \mathcal{R}^{i}{ }_{j} \mathcal{R}^{j}{ }_{i}, \\
\mathcal{V}_{z=3}:= & g_{4} \mathcal{R}^{3}+g_{5} \mathcal{R} \mathcal{R}^{i}{ }_{j} \mathcal{R}^{j}{ }_{i}+g_{6} \mathcal{R}^{i}{ }_{j} \mathcal{R}^{j}{ }_{k} \mathcal{R}^{k}{ }_{i} \\
& +g_{7} \mathcal{R} \nabla^{2} \mathcal{R}+g_{8} \nabla_{i} \mathcal{R}_{j k} \nabla^{i} \mathcal{R}^{j k},
\end{aligned}
$$

where $\Lambda$ is the cosmological constant and $\lambda$ and $g_{n}$ ( $n=1-8)$ are dimensionless coupling constants. The potential terms include the higher-order spatial curvatures $\mathcal{R}_{i j}$, $\mathcal{R}:=\mathcal{R}_{i}^{i}$ up to sixth-order spatial derivatives. In what follows, we adopt the unit $m_{\mathrm{LV}}=1$ unless otherwise noted.

We shall focus on the FLRW spacetime whose induced metric $g_{i j}$ is given by

$$
\begin{aligned}
d s^{2} & =g_{i j} d x^{i} d x^{j} \\
& =a^{2}\left[d \chi^{2}+f(\chi)^{2}\left(d \theta^{2}+\sin ^{2} \theta d \phi^{2}\right)\right]
\end{aligned}
$$

with

$$
f(\chi):= \begin{cases}\chi & \text { for } K=0 \\ \sin \chi & \text { for } K=1 \\ \sinh \chi & \text { for } K=-1\end{cases}
$$

where $a$ is a scale factor which depends only on the time. The metrics whose spatial curvature $K=0,1$, and -1 correspond to flat, closed, and open FLRW spacetime, respectively. The domains of the variables are defined by

\footnotetext{
${ }^{1}$ In the context of Einstein-aether gravity as an infrared effective theory of HL gravity, the Lorentz-violating mass scale $m_{\mathrm{LV}}=\left(8 \pi G_{\mathrm{LV}}\right)^{-1}$ can be related with ordinal Newton constant $G_{\mathrm{N}}$ as $G_{\mathrm{N}}=\left(-g_{1}\right) G_{\mathrm{LV}}$ by taking the weak gravity limit [23].
} 


$$
\begin{cases}0 \leq \chi<\infty & \text { for } K=0,-1 \\ 0 \leq \chi<\pi & \text { for } K=1\end{cases}
$$

$0 \leq \theta<\pi$, and $0 \leq \phi<2 \pi$.

Assuming the above ansatz and taking a variation with respect to $a, N$, and $N_{i}$, we obtain the dynamical equation of scale factor, the Hamiltonian constraint, and the momentum constraint, respectively. Since the momentum constraint gives a trivial relation, we omit it. The dynamical equation of the scale factor is given by

$$
\begin{aligned}
& \frac{3 \lambda-1}{2}\left(2 \dot{H}+3 H^{2}\right) \\
& \quad-\left(\Lambda+g_{1} \frac{K}{a^{2}}-\frac{g_{r}}{3 a^{4}}-\frac{g_{s}}{a^{6}}\right)=0,
\end{aligned}
$$

where $H:=\partial_{t} a / a=\dot{a} / a$ is a Hubble parameter, $\dot{H}=\partial_{t} H$, and

$$
\begin{gathered}
g_{r}:=6 K^{2}\left(3 g_{2}+g_{3}\right), \\
g_{s}:=12 K^{3}\left(9 g_{4}+3 g_{5}+g_{6}\right) .
\end{gathered}
$$

After taking the variation, we have imposed the gauge condition so that $N=1$ and $N_{i}=0$. It is notable that the terms derived from the higher spatial curvatures behave as virtual matter fields. More specifically, the $g_{r}$ and $g_{s}$ terms effectively work as "radiation" and "stiff matter," respectively. Note that the $g_{7}$ and $g_{8}$ terms do not affect the background solution because of the spacetime symmetry.

We turn our attention to the Friedmann equation, which corresponds to the Hamiltonian constraint in FLRW spacetime. In our case, we cannot construct the Friedmann equation via taking a variation of the action. Since we have imposed the projectability condition, i.e., $N(t, x) \rightarrow N(t)$, the Hamiltonian constraint takes the following form:

$$
\int d^{3} x\left(\mathcal{L}_{K}-\mathcal{L}_{P}\right)=0
$$

namely, the Hamiltonian constraint turns out to be a global condition instead of a local one. It means that we have to know the information within the entire spacetime to construct the Hamiltonian constraint.

If we assume the complete isotropy and homogeneity of the universe, a Friedmann-like equation can be derived via the integration of the dynamical equation for the scale factor (and matter conservation law) as follows:

$$
H^{2}-\frac{2}{3(3 \lambda-1)}\left[\Lambda+3 g_{1} \frac{K}{a^{2}}+\frac{g_{r}}{a^{4}}+\frac{g_{s}}{a^{6}}\right]=\frac{\mathcal{C}}{a^{3}},
$$

where $\mathcal{C}$ is an integration constant which behaves as a dustlike component. Of course, the complete isotropy and homogeneity of our Universe is not trivial, because the area where we can observe is inside the cosmological horizon. It is possible that $\mathcal{C}$ may depend on the spatial coordinate $x^{i}$ on the scale beyond the cosmological horizon. Then, $\mathcal{C}$ must be distributed so that the global Hamiltonian constraint (2.12) is satisfied, which may represent dark matter [24]. In the rest of this paper, we assume the background geometry possesses a complete isotropy and homogeneity, and, thus, $\mathcal{C}$ is treated as a constant.

For later convenience, we define the following quantities:

$$
\begin{aligned}
\mathcal{E}:= & (3 \lambda-1)\left(2 \dot{H}+3 H^{2}\right) \\
& -2\left(\Lambda+g_{1} \frac{K}{a^{2}}-\frac{g_{r}}{3 a^{4}}-\frac{g_{s}}{a^{6}}\right),
\end{aligned}
$$

$\mathcal{H}:=6(3 \lambda-1) H^{2}-4\left[\Lambda+3 g_{1} \frac{K}{a^{2}}+\frac{g_{r}}{a^{4}}+\frac{g_{s}}{a^{6}}\right]$.

Then, the dynamical equation of the scale factor and the Friedmann-like equation can be written by $\mathcal{E}=0$ and $\mathcal{H}=6(3 \lambda-1) \mathcal{C} / a^{3}$, respectively. Since $\mathcal{C}$ does not depend on the time, the value of $\mathcal{H}$ is determined by the initial condition.

One may notice that the spacetime dynamics for $\lambda>1 / 3$ and $\lambda<1 / 3$ are completely different, i.e., the sign of time derivative terms are flipped. Since the limit to GR can be obtained by taking $\lambda \rightarrow+1$, we exclude the $\lambda \leq 1 / 3$ case in what follows. Additionally, the value of $g_{1}$ must be negative to recover the result based on GR at least at a background level. Then, we set $g_{1}=-1$ in the rest part of this paper by performing a suitable rescaling of the time.

\section{PERTURBATION ANALYSIS AROUND A NONFLAT FLRW BACKGROUND}

Since the Arnowitt-Deser-Misner (ADM) formalism is employed in this paper, we define the perturbed quantities of ADM variables $\delta N, \delta N_{i}$, and $\delta g_{i j}$ as follows:

$$
\begin{gathered}
N=\bar{N}+\delta N, \\
N_{i}=\bar{N}_{i}+\delta N_{i}, \\
g_{i j}=\bar{g}_{i j}+\delta g_{i j},
\end{gathered}
$$

where $\bar{N}, \bar{N}_{i}$, and $\bar{g}_{i j}$ denote the background lapse function, shift vector, and three-dimensional induced metric, respectively. Since we consider the quadratic gravitational action, we define $\delta N, \delta N_{i}$, and $\delta g_{i j}$ as follows: 


$$
\begin{aligned}
\delta N & =\bar{N}\left[\frac{\alpha}{\bar{N}}+\frac{1}{2}\left(\frac{\alpha}{\bar{N}}\right)^{2}\right], \\
\delta N_{i} & =\beta_{i}, \\
\delta g_{i j} & =h_{i j}+\frac{1}{2} \bar{g}^{a b} h_{a i} h_{b j},
\end{aligned}
$$

where $\alpha, \beta_{i}$, and $h_{i j}$ are perturbations of first order. Furthermore, we define the perturbed quantities with upper indices as $h_{j}^{i}:=\bar{g}^{i a} h_{a j}, h^{i j}:=\bar{g}^{i a} \bar{g}^{j b} h_{a b}, h:=\bar{g}^{a b} h_{a b}$, and $\beta^{i}:=\bar{g}^{i a} \beta_{a}$. Then, we shall decompose $\alpha, \beta_{i}$, and $h_{i j}$ into the scalar, vector, and tensor modes, respectively.

\section{A. Decomposition of perturbation}

To decompose each perturbation mode into the scalar, vector, and tensor ones, we employ tensor harmonic functions. In particular, in a closed and open FLRW background, spherical or pseudospherical harmonics are applied, respectively [25-27]. More specifically, $(\chi, \theta, \phi)$ dependences of perturbed ADM variables can be expanded by each mode of harmonics, which is similar to the black hole perturbation theory. For example, a scalar function $\Theta$ can be expanded by scalar harmonics $Q^{(n ; l m)}$ in FLRW spacetime $^{2}$ :

$\Theta(t, \chi, \theta, \phi)=\sum_{n} \sum_{l} \sum_{m=-l}^{l} \Theta^{(n ; l m)}(t) Q^{(n ; l m)}(\chi, \theta, \phi)$,

where $\Theta^{(n ; l m)}$ is a coefficient of each $(n ; l, m)$ mode which depends only on the time. We summarize the definitions of the tensor spherical and pseudospherical harmonics in Appendix A and useful formulas in Appendix B. Although we do not discuss the case with $K=0$ in those parts, the harmonic function in flat FLRW spacetime can be obtained by replacing the function $X^{(n ; l)}$ which is the $\chi$-dependent part into the spherical Bessel function.

Since we consider the four-dimensional spacetime, ten types of independent tensor harmonics must be equipped as a basis set. In this paper, we employ one possible orthonormal basis set $\mathbf{Y}$ as follows:

$$
\begin{aligned}
\mathbf{Y}= & \left\{Q, Q_{i}, Q_{i j}, P_{i j}, S_{(o) i}, S_{(e) i}, S_{(o) i j}, S_{(e) i j},\right. \\
& \left.G_{(o) i j}, G_{(e) i j}\right\} .
\end{aligned}
$$

We turn our attention to the decomposition of ADM variables by harmonics. The scalar perturbation can be expanded by the scalar harmonics:

\footnotetext{
${ }^{2}$ For simplicity, we denote $\sum$ to sum up $n$ modes. To be precise, it must be replaced by integration symbol $\int$ if we consider a flat and open FLRW spacetime, because $n$ turns to be a continuous number.
}

$$
\begin{gathered}
\alpha^{(\text {scalar })}=\alpha(t), \\
\beta_{i}^{\text {(scalar })}=\sum_{n, l, m} a^{2}\left[\beta_{(Q)}^{(n ; l m)} Q_{i}^{(n ; l m)}\right], \\
h_{i j}^{(\text {scalar })}=\sum_{n, l, m} a^{2}\left[h_{(Q)}^{(n ; l m)} Q_{i j}^{(n ; l m)}+h_{(P)}^{(n ; l m)} P_{i j}^{(n ; l m)}\right] .
\end{gathered}
$$

Note that we do not have to expand the perturbed lapse function in terms of the harmonic function. Since the projectability condition is imposed, the lapse perturbation also depends only on the time variable. The vector perturbation can be expanded by the vector harmonics:

$$
\begin{aligned}
& \beta_{i}^{(\text {vector })}=\sum_{n, l, m} a^{2}\left[\beta_{(S ; o)}^{(n ; l m)} S_{(o) i}^{(n ; l m)}+\beta_{(S ; e)}^{(n ; l m)} S_{(e) i}^{(n ; l m)}\right], \\
& h_{i j}^{(\text {vector })}=\sum_{n, l, m} a^{2}\left[h_{(S ; o)}^{(n ; l m)} S_{(o) i j}^{(n ; l m)}+h_{(S ; e)}^{(n ; l m)} S_{(e) i j}^{(n ; l m)}\right] .
\end{aligned}
$$

The lapse function is not perturbed by the vector perturbation. The tensor perturbation can be expanded by the tensor harmonics:

$h_{i j}^{\text {(tensor) }}=\sum_{n, l, m} a^{2}\left[h_{(G ; o)}^{(n ; l m)} G_{(o) i j}^{(n ; l m)}+h_{(G ; e)}^{(n ; l m)} G_{(e) i j}^{(n ; l m)}\right]$.

Note that the lapse and shift perturbation do not exist in the tensor perturbation. In this paper, we eliminate the following perturbations by choosing the gauge:

$$
\alpha=h_{(P)}^{(n ; l m)}=h_{(S ; o)}^{(n ; l m)}=h_{(S ; e)}^{(n ; l m)}=0 .
$$

In Appendix C, the gauge structure in FLRW spacetime is summarized.

\section{B. Quadratic action}

We turn our attention to the perturbed action at quadratic order by employing harmonics. Before performing a perturbation analysis, we shall mention the $n=1$ case for closed FLRW. In this case, the perturbation is given by

$$
\beta_{i}^{(1 ; 00)}=0, \quad h_{i j}^{(1 ; 00)}=\frac{1}{\sqrt{2} \pi} h_{(Q)}^{(1 ; 00)} \bar{g}_{i j} .
$$

One notice that this mode corresponds to just a shift of the scale factor, i.e., $a(t) \rightarrow a(t)+\delta a(t)$, and, thus, we exclude this perturbation mode in what follows.

We first consider the perturbation of kinetic terms to clarify the dynamical degree of freedom. The quadratic kinetic actions for scalar, vector, and tensor perturbation are given, respectively, by 


$$
\begin{aligned}
& \delta_{(2)} \mathcal{L}_{K}^{(\text {scalar })}= a^{3}\left[-\frac{1}{2}(3 \lambda-1) \dot{h}_{(Q)}^{2}\right. \\
&+\frac{3}{4}(3 \lambda-1)\left(2 \dot{H}+3 H^{2}\right) h_{(Q)}^{2} \\
&-2(3 \lambda-1) \frac{\nu}{\sqrt{3}} \dot{h}_{(Q)} \beta_{(Q)} \\
&\left.-2\left[(\lambda-1) \nu^{2}+2 K\right] \beta_{(Q)}^{2}\right], \\
& \delta_{(2)} \mathcal{L}_{K}^{(\text {vector })}=a^{3}\left(\nu^{2}-3 K\right) \beta_{(S)}^{2}, \\
& \delta_{(2)} \mathcal{L}_{K}^{(\text {tensor })}=\frac{a^{3}}{2} \dot{h}_{(G)}^{2},
\end{aligned}
$$

where $\nu^{2}$ is an eigenvalue of the harmonics which is defined in terms of the perturbation mode $n \geq 1$ :

$$
\nu^{2}:=\left\{\begin{array}{lll}
n^{2}-1, & n \in \mathbb{R} & \text { for } K=0, \\
n^{2}-1, & n \in \mathbb{N} & \text { for } K=1, \\
n^{2}+1, & n \in \mathbb{R} & \text { for } K=-1 .
\end{array}\right.
$$

Since the perturbations with different degrees do not mix, we have abbreviated the superscript $(n ; l m)$. Note that $\beta_{(S)}$ and $h_{(G)}$ vanish when $l=0$ and $l \leq 1$, respectively.

Taking a variation with respect to $\beta_{(Q)}$ and $\beta_{(S)}$, we obtain the following constraint equations:

$$
\begin{aligned}
& 0=(3 \lambda-1) \frac{\nu}{\sqrt{3}} \dot{h}_{(Q)}+2\left[(\lambda-1) \nu^{2}+2 K\right] \beta_{(Q)}, \\
& 0=2\left(\nu^{2}-3 K\right) \beta_{(S)},
\end{aligned}
$$

and, plugging these relations into the actions, we obtain the simplified quadratic action as follows:

$$
\begin{aligned}
\delta_{(2)} \mathcal{L}_{K}^{\text {(scalar })}= & a^{3}\left[\frac{(3 \lambda-1)\left(\nu^{2}-3 K\right)}{3\left[(\lambda-1) \nu^{2}+2 K\right]} \dot{h}_{(Q)}^{2}\right. \\
& \left.+\frac{3}{4}(3 \lambda-1)\left(2 \dot{H}+3 H^{2}\right) h_{(Q)}^{2}\right],
\end{aligned}
$$

and the vector mode vanishes. Summing up the quadratic potential terms, we finally obtain

$$
\begin{aligned}
& \delta_{(2)} \mathcal{L}^{\text {(tensor) }}=\frac{a^{3}}{2}\left[\mathcal{F}_{(G)} \dot{h}_{(G)}^{2}-\mathcal{G}_{(G)} h_{(G)}^{2}\right], \\
& \delta_{(2)} \mathcal{L}^{(\text {scalar })}=\frac{a^{3}}{2}\left[\mathcal{F}_{(Q)} \dot{h}_{(Q)}^{2}-\mathcal{G}_{(Q)} h_{(Q)}^{2}\right],
\end{aligned}
$$

where $\mathcal{F}_{(G)}$ and $\mathcal{G}_{(G)}$ can be regarded as the kinetic term and the mass term of the tensor perturbation, respectively, which are given by

$$
\begin{aligned}
\mathcal{F}_{(G)}: & =1, \\
\mathcal{G}_{(G)}= & \frac{\nu^{2}}{a^{2}}+\frac{\nu^{2}}{3 a^{4}}\left[-\frac{2 g_{r}}{K}+3 g_{3} \nu^{2}\right] \\
& +\frac{\nu^{2}}{a^{6}}\left[-\frac{g_{s}}{K}+6 g_{56} K \nu^{2}+g_{8} \nu^{2}\left(\nu^{2}-2 K\right)\right],
\end{aligned}
$$

where $g_{56}:=g_{5}+g_{6}$. On the other hand, $\mathcal{F}_{(Q)}$ and $\mathcal{G}_{(Q)}$ are regarded as the kinetic term and the mass term of the scalar perturbation:

$$
\begin{aligned}
\mathcal{F}_{(Q)}:= & \frac{2(3 \lambda-1)\left(\nu^{2}-3 K\right)}{3\left[(\lambda-1) \nu^{2}+2 K\right]} \\
\mathcal{G}_{(Q)}:= & -\frac{2}{3 a^{2}}\left(\nu^{2}-3 K\right) \\
& +\frac{2}{27 a^{4}}\left(\nu^{2}-3 K\right)\left[\frac{2 g_{r}}{K^{2}}\left(2 \nu^{2}-3 K\right)+3 g_{3} \nu^{2}\right] \\
& +\frac{2}{9 a^{6}}\left(\nu^{2}-3 K\right)\left[\frac{g_{s}}{K^{2}}\left(4 \nu^{2}-9 K\right)\right. \\
& +2\left(3 g_{56}-4 g_{7}\right) K \nu^{2} \\
& \left.+\left(-8 g_{7}+3 g_{8}\right) \nu^{2}\left(3 \nu^{2}-10 K\right)\right] .
\end{aligned}
$$

The terms including Hubble parameter $H$ and its time derivative $\dot{H}$ have been eliminated by applying the background equation of motion $\mathcal{E}=0$. Note that there does not exist an integration constant $\mathcal{C}$ in the Friedmannlike equation $\mathcal{H}=6(3 \lambda-1) \mathcal{C} / a^{3}$. It is because the Hamiltonian constant basically arises as a coefficient of the lapse perturbation. In our case, we have fixed the gauge so that $\alpha=0$; then, there is no ambiguity in the quadratic action. Additionally, one notices that there are no dynamical scalar modes in the closed FLRW spacetime with $n=2$, because $\mathcal{F}_{(Q)}$ automatically vanishes.

Particular attention should be given to the fact that the quadratic actions (3.22) and (3.23) cannot be reduced into the result based on GR even if we take the limit $\lambda \rightarrow+1$ and the terms from the higher spatial curvature are neglected. Based on GR, the scalar degree of freedom is absent if we consider the vacuum FLRW spacetime. However, in our case, the scalar perturbations cannot be eliminated when we take such a limit. This fact is due to the gauge structure shown in Appendix C. More specifically, the time derivatives of scalar perturbation $\dot{h}_{(Q)}$ must appear because of the Lorentz violation; i.e., the rotation of the time direction is not allowed.

In order to clarify the stability conditions for the perturbations, we first focus on the coefficient of kinetic terms and mass terms. 


\section{Ghost avoidance}

We shall concentrate on the terms derived from $\mathcal{L}_{K}$. Since $\mathcal{F}_{(G)}$ and $\mathcal{F}_{(Q)}$ are the coefficients of the kinetic terms of each perturbation mode, the conditions $\mathcal{F}_{(G)}>0$ and $\mathcal{F}_{(Q)}>0$ are required to avoid ghost instabilities in the tensor and scalar perturbation, respectively. Since $\mathcal{F}_{(G)}=1$, the tensor perturbations do not show ghost instability for any choice of the coupling constants.

On the other hand, the condition for the scalar one is not trivial. In flat FLRW spacetime, the coefficient of the kinetic term is reduced into $\mathcal{F}_{(Q)}=2(3 \lambda-1) / 3(\lambda-1)$. Then, one can see that the limit $\lambda \rightarrow+1$ is singular. In order to satisfy the positivity of $\mathcal{F}_{(Q)}$, we require

$$
\lambda>1,
$$

which coincides with the ghost-free condition in Minkowski spacetime [14]. In closed FLRW spacetime, namely, $K=1$, to satisfy $\mathcal{F}_{(Q)}>0$ for every $n \geq 3$, we find

$$
\lambda \geq 1
$$

Because of the nonzero spatial curvature $K$ in the denominator of (3.26), we do not find any singular behavior in the limit $\lambda \rightarrow+1$ unlike the flat case. In open FLRW spacetime, namely, $K=-1$, the stability condition for every $n \geq 1$ mode is given by

$$
\lambda>2,
$$

which gives a tighter condition than that of the closed case.

\section{Tachyon avoidance}

The terms derived from $\mathcal{L}_{P}$, namely, $\mathcal{G}_{(G)}$ and $\mathcal{G}_{(Q)}$, can be associated with squared effective masses of tensor and scalar perturbations, respectively [see (4.15)]. Therefore, $\mathcal{G}_{(G)} \geq 0$ and $\mathcal{G}_{(Q)} \geq 0$ must be satisfied; otherwise, tachyon instability appears. One can see that equalities $\mathcal{G}_{(G)}=0$ and $\mathcal{G}_{(Q)}=0$ give quadratic equations with respect to $a^{2}$ and $\nu^{2}$ with the coefficients related with $g_{i}$. It means that the ranges for the scale factor in which $\mathcal{G}_{(G)} \geq 0$ and $\mathcal{G}_{(Q)} \geq 0$ can be expressed in terms of the coupling constants, in principle.

In this part, we focus on the infrared stability; i.e., the case without effects of higher-order spatial curvatures is considered. Then, the mass terms in the quadratic action are reduced into

$$
\mathcal{G}_{(Q)} \approx-\frac{2}{3 a^{2}}\left(\nu^{2}-3 K\right), \quad \mathcal{G}_{(G)} \approx \frac{\nu^{2}}{a^{2}} .
$$

We see the tensor perturbation mode shows a positive squared effective mass, while the scalar one possesses the opposite sign for any spatial curvature $K$. Thus, in the infrared regime, the negative squared effective masses of the scalar perturbations cannot be avoided. The similar situation has been found in a perturbation around Minkowski spacetime [17,18]. Of course, the negative squared effective mass does not always lead to tachyon instability. If the growing time scale for the scalar perturbation is sufficiently small relative to the cosmological time scale, the instability is suppressed.

Another possibility to avoid infrared tachyon instabilities is to consider the extended version of the HL theory, namely, relaxing the projectability condition [18]. It is known that an additional $\left(\nabla_{i} \ln N\right)^{2}$ term in extended action can stabilize the scalar perturbation at least in a flat background.

\section{STABILITY ANALYSIS OF SINGULARITY-FREE SOLUTIONS}

In this section, we analyze the stabilities of singularityfree solutions in FLRW spacetime. Note that we cannot find any singularity-free solution if $K=0$, because the threeRicci curvature $\mathcal{R}_{i j}$ vanishes at the background level. More specifically, there is no effective matter component to violate the null energy condition. Therefore, we limit our analysis to the case with $K= \pm 1$.

Since the typical scale of the singularity avoidance is expected to be the Planck scale, we focus only on the solutions which potentially connect to a macroscopic universe. More specifically, we consider bouncing cosmological solutions with a positive cosmological constant, which show bouncing behavior at $a=a_{T}>0$ and turn to an accelerating expanding phase.

One may consider that the bouncing solution without a cosmological constant in open FLRW spacetime can also evolve to a macroscopic universe with asymptotic Milne expansion. However, such solutions seem to be unstable because of weak Hubble friction during the expanding phase. As we mentioned previously, the scalar perturbation possesses negative $\mathcal{G}_{(Q)}$ in the infrared regime, and thus, we exclude the case with a nonpositive cosmological constant.

\section{A. Background solutions}

The classification of the solutions in a vacuum FLRW background is performed in Ref. [14]. In that paper, it is found that there are two types of singularity-free solutions. One is a bouncing universe; that is, the initial contracting universe turns into the expanding phase at $a=a_{T}$, and the universe keeps expanding without a finite upper bound of the scale factor. The other is an oscillating universe whose scale factor is bounded in the range of $0<a_{\min } \leq$ $a \leq a_{\max }<\infty$. Then, the universe shows periodic oscillatory behavior without a singularity.

The dynamics of the background spacetime can be examined via a rewritten Friedmann-like equation:

$$
\frac{1}{2} \dot{a}^{2}+\mathcal{U}(a)=0,
$$


with

$$
\mathcal{U}(a)=\frac{1}{3 \lambda-1}\left[K-\frac{\Lambda}{3} a^{2}-\frac{g_{r}}{3 a^{2}}-\frac{g_{s}}{3 a^{4}}\right] .
$$

For simplicity, we have taken the integration constant $\mathcal{C}=0$. Since the first term of the left-hand side of (4.1) is not negative, the possible ranges of the scale factor are where $\mathcal{U} \leq 0$. Note that $g_{r}$ and $g_{s}$ are related with the coupling constants in $\mathcal{L}_{P}$, and then these values can take both a plus and minus sign if the coupling constants are arbitrary. Therefore, we can consider the situation in which the scale factor is bounded below by some nonzero minimum value. It means the universe is forbidden to fall down into the singularity. We would like to stress that the singularity avoidance is induced because the energy condition is effectively violated.

To analyze the background dynamics with $\Lambda>0$, it is convenient to rewrite the potential $\mathcal{U}$ by rescaled variables with respect to $\ell:=\sqrt{3 / \Lambda}$ :

$$
\tilde{\mathcal{U}}(\tilde{a})=\frac{1}{3 \lambda-1}\left[K-\tilde{a}^{2}-\frac{\tilde{g}_{r}}{3 \tilde{a}^{2}}-\frac{\tilde{g}_{s}}{3 \tilde{a}^{4}}\right],
$$

where $\tilde{a}:=a / \ell, \tilde{g}_{r}:=g_{r} / \ell^{2}$, and $\tilde{g}_{s}:=g_{s} / \ell^{4}$. Since $\tilde{\mathcal{U}}=0$ is essentially a cubic equation of $\tilde{a}^{2}$, we obtain three analytic solutions as follows:

$$
\left(\tilde{a}_{I}^{[K]}\right)^{2}:=\frac{1}{6}\left[2 K+\frac{4\left(K^{2}-\tilde{g}_{r}\right)}{\tilde{\xi}_{I}^{[K]}}+\tilde{\xi}_{I}^{[K]}\right],
$$

with

$$
\begin{aligned}
\tilde{\xi}_{I}^{[K]}:= & \mathrm{pv} 2^{2 / 3}\left(e^{2 \pi i / 3}\right)^{I}\left[2 K^{3}-3 \tilde{g}_{r} K-9 \tilde{g}_{s}\right. \\
& \left.+9 \sqrt{\left(\tilde{g}_{s}-\tilde{g}_{s}^{[K](+)}\right)\left(\tilde{g}_{s}-\tilde{g}_{s}^{[K](-)}\right)}\right]^{1 / 3}, \\
\tilde{g}_{s}^{[K]( \pm)}:= & \frac{1}{9}\left[2 K^{3}-3 \tilde{g}_{r} K \pm 2\left(K^{2}-\tilde{g}_{r}\right)^{3 / 2}\right],
\end{aligned}
$$

where $I=1,2,3$. Note that the points at which $\tilde{\mathcal{U}}(\tilde{a})=0$ can be found when corresponding $\tilde{a}_{I}^{[K]}$ takes real and positive values.

Additionally, the above roots cannot be applied to the special case with $\tilde{g}_{s}=0$. In this instance, we obtain two analytic solutions as follows:

$$
\left(\tilde{a}_{ \pm}^{[K]}\right)^{2}:=\frac{1}{2}\left[K \pm \sqrt{K^{2}-\frac{4 \tilde{g}_{r}}{3}}\right] .
$$

\section{Closed FLRW $(K=1)$}

In a closed FLRW universe, namely, the $K=1$ case, three types of singularity-free solutions are found. We show the typical potentials $\tilde{\mathcal{U}}$ for these solutions in Fig. 1 .

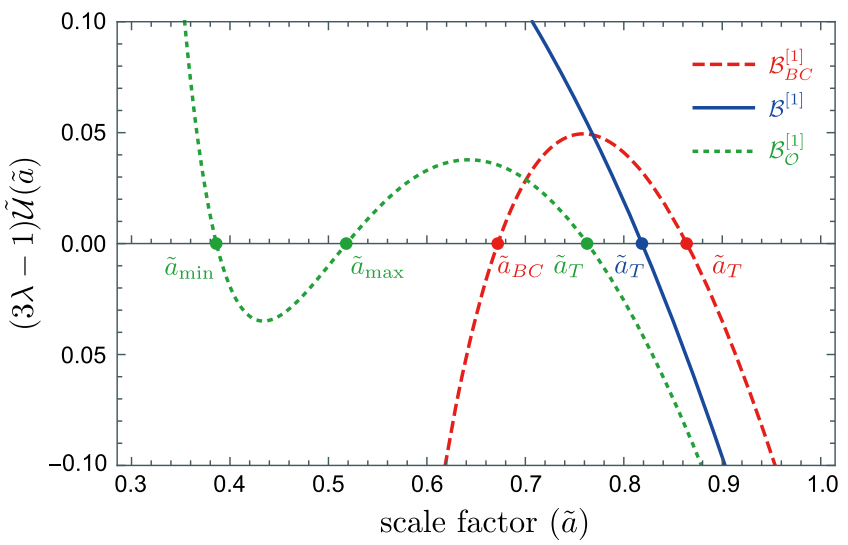

FIG. 1. The potentials $\tilde{\mathcal{U}}(\tilde{a})$ for $\mathcal{B}_{B C}^{[1]}$ (red dashed curve), $\mathcal{B}^{[1]}$ (blue solid curve), and $\mathcal{B}_{\mathcal{O}}^{[1]}$ (green dotted curve). The coupling constants are chosen as $\tilde{g}_{r}=3 / 10$ and $\tilde{g}_{s}=2 / 10$ for $\mathcal{B}_{B C}^{[1]}, \tilde{g}_{r}=$ $17 / 20$ and $\tilde{g}_{s}=-1 / 8$ for $\mathcal{B}^{[1]}$, and $\tilde{g}_{r}=17 / 20$ and $\tilde{g}_{s}=-7 / 100$ for $\mathcal{B}_{\mathcal{O}}^{[1]}$. The bouncing radii and the maximum radii of the big crunch solution are denoted by $\tilde{a}_{T}$ and $\tilde{a}_{B C}$, respectively. Additionally, the maximum and minimum radii of oscillation are given by $\tilde{a}_{\max }$ and $\tilde{a}_{\min }$, respectively.

(a) $\mathcal{B}_{B C}^{[1]} \cdot-\mathrm{A}$ universe which shows bouncing behavior for initial scale factor $\tilde{a}_{\text {ini }} \geq \tilde{a}_{T}$, however, evolves into a big crunch for $\tilde{a}_{\text {ini }} \leq \tilde{a}_{B C}$. We classify this type of solution as $\mathcal{B}_{B C}^{[1]}$. The typical potential is given by the dashed red curve in Fig. 1. Note that the domain $\tilde{a}_{B C}<\tilde{a}<\tilde{a}_{T}$ is forbidden. The solutions $\mathcal{B}_{B C}^{[1]}$ can be found in the following two cases:

$$
\begin{aligned}
& \text { (i) } 0<\tilde{g}_{s}<\tilde{g}_{s}^{[1](+)} \\
& \text { with } \quad \tilde{a}_{B C}=\tilde{a}_{2}^{[1]}, \quad \tilde{a}_{T}=\tilde{a}_{3}^{[1]} . \\
& \text { (ii) } \tilde{g}_{s}=0, \quad 0<\tilde{g}_{r}<\frac{3}{4} \\
& \text { with } \quad \tilde{a}_{B C}=\tilde{a}_{-}^{[1]}, \quad \tilde{a}_{T}=\tilde{a}_{+}^{[1]} .
\end{aligned}
$$

(b) $\mathcal{B}^{[1]}$ - $\mathrm{A}$ universe which bounces at $\tilde{a}=\tilde{a}_{T}$ without a big-bang singularity for any possible initial scale factor $\tilde{a}_{\text {ini }} \geq \tilde{a}_{T}$. We classify this type of solution as $\mathcal{B}^{[1]}$. The typical potential is given by the solid blue curve in Fig. 1. The solutions $\mathcal{B}^{[1]}$ can be found in the following three cases:

(i) $\tilde{g}_{s}^{[1](+)}<\tilde{g}_{s}<0, \quad \tilde{g}_{r}<1 \quad$ with $\quad \tilde{a}_{T}=\tilde{a}_{1}^{[1]}$.

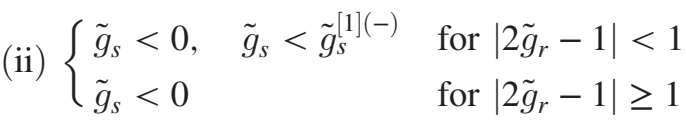

with $\quad \tilde{a}_{T}=\tilde{a}_{3}^{[1]}$.

(iii) $\tilde{g}_{s}=0, \quad \tilde{g}_{r} \leq 0 \quad$ with $\quad \tilde{a}_{T}=\tilde{a}_{+}^{[1]}$. 
(c) $\mathcal{B}_{\mathcal{O}}^{[1]} \cdot-\mathrm{A}$ universe which shows bouncing behavior for initial scale factor $\tilde{a}_{\text {ini }} \geq \tilde{a}_{T}$, on the other hand, oscillates if $\tilde{a}_{\min } \leq \tilde{a}_{\text {ini }} \leq \tilde{a}_{\max }$. The other choice of the initial scale factor is forbidden. We classify this type of solution as $\mathcal{B}_{\mathcal{O}}^{[1]}$. The typical potential is given by the dotted green curve in Fig. 1. The solutions $\mathcal{B}_{\mathcal{O}}^{[1]}$ can be found if the following condition is satisfied:

$$
\begin{array}{cccc}
\tilde{g}_{s} & <0, & \tilde{g}_{s}^{[1](-)}<\tilde{g}_{s}<\tilde{g}_{s}^{[1](+)} \quad \text { with } \\
\tilde{a}_{\min } & =\tilde{a}_{1}^{[1]}, & \tilde{a}_{\max }=\tilde{a}_{2}^{[1]}, & \tilde{a}_{T}=\tilde{a}_{3}^{[1]} .
\end{array}
$$

\section{Open FLRW $(K=-1)$}

In open FLRW, namely, the $K=-1$ case, two types of singularity-free solutions are found. We show the typical potentials $\tilde{\mathcal{U}}$ for these solutions in Fig. 2 .

(a) $\mathcal{B}_{B C}^{[-1]}$. - The properties of this solution are quite similar to those of $\mathcal{B}_{B C}^{[1]}$ in the closed case. Namely, this type of solution shows bouncing behavior for initial scale factor $\tilde{a}_{\text {ini }} \geq \tilde{a}_{T}$, but, on the other hand, evolves into a big-bang singularity for $\tilde{a}_{\text {ini }} \leq \tilde{a}_{B C}$. We classify this type of solution as $\mathcal{B}_{B C}^{[-1]}$. The typical potential is given by the dashed red curve in Fig. 2. The solutions $\mathcal{B}_{B C}^{[-1]}$ can be found if the following condition is satisfied:

$$
\begin{aligned}
0 & <\tilde{g}_{s}<\tilde{g}_{s}^{[-1](+)}, \quad \tilde{g}_{r}<0 \quad \text { with } \\
\tilde{a}_{B C} & =\tilde{a}_{2}^{[-1]}, \quad \tilde{a}_{T}=\tilde{a}_{3}^{[-1]} .
\end{aligned}
$$

(b) $\mathcal{B}^{[-1]}$. - As is the case with $\mathcal{B}^{[1]}$ in closed FLRW, this type of solution also shows bouncing behavior for any

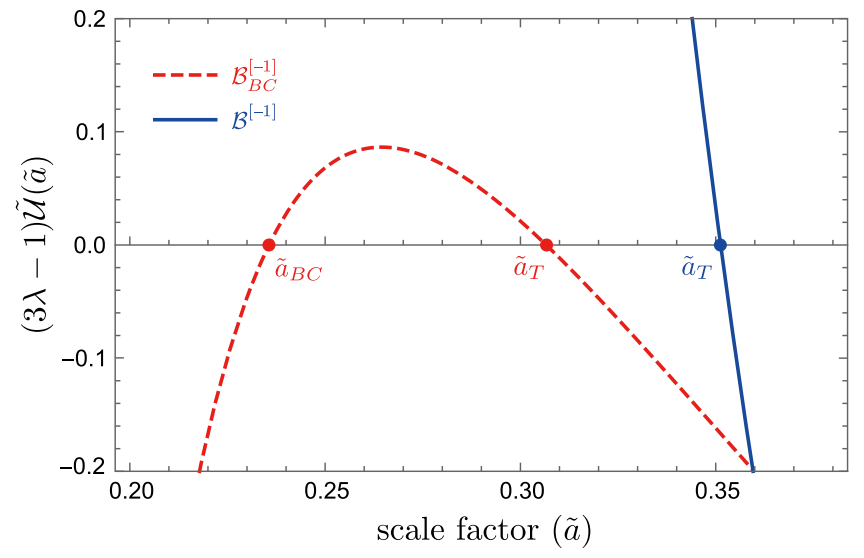

FIG. 2. The potentials $\tilde{\mathcal{U}}(\tilde{a})$ for $\mathcal{B}_{B C}^{[-1]}$ (red dashed curve) and $\mathcal{B}^{[-1]}$ (blue solid curve) in an open FLRW universe. The coupling constants are chosen as $\tilde{g}_{r}=-1 / 2$ and $\tilde{g}_{s}=9 / 500$ for $\mathcal{B}_{B C}^{[-1]}$ and $\tilde{g}_{r}=4 / 5$ and $\tilde{g}_{s}=-3 / 20$ for $\mathcal{B}^{[-1]}$. The bouncing radii and the maximum radii of the big crunch solution are denoted by $\tilde{a}_{T}$ and $\tilde{a}_{B C}$, respectively. possible initial scale factor $\tilde{a}_{\text {ini }} \geq \tilde{a}_{T}$. We classify this type of solution as $\mathcal{B}^{[-1]}$. The typical potential is given by the solid blue curve in Fig. 2. The solutions $\mathcal{B}_{B C}^{[-1]}$ can be found in the following two cases:

$$
\begin{aligned}
& \text { (i) } \tilde{g}_{s}<0 \quad \text { with } \tilde{a}_{T}=\tilde{a}_{3}^{[-1]} . \\
& \text { (ii) } \tilde{g}_{s}=0, \quad \tilde{g}_{r}<0 \quad \text { with } \quad \tilde{a}_{T}=\tilde{a}_{+}^{[-1]} .
\end{aligned}
$$

We show the properties of singularity-free solutions in Table I and the distribution of the singularity-free solutions in the $\left(\tilde{g}_{r}, \tilde{g}_{s}\right)$ plane in Fig. 3.

\section{B. Perturbation analysis}

We turn our attention to the ultraviolet stability. Since the conditions for avoiding ghost instabilities have been discussed in the previous section, we concentrate on the positivities of $\mathcal{G}_{(Q)}$ and $\mathcal{G}_{(G)}$ including higher-order spatial curvatures. Our aim is to clarify the stability conditions for all perturbation modes with finite values of the coupling constants $\lambda$ and $g_{i}(i=1-8)$. Otherwise, the asymptotic safety is violated, which may cause the divergence of the gravitational force in the ultraviolet regime.

\section{Tensor perturbations}

The stability condition for the tensor perturbation is given by $\mathcal{G}_{(G)} \geq 0$. The positivity of $\mathcal{F}_{(G)}$ is automatically satisfied; namely, there is no ghost tensor mode for any choice of coupling constants. Since $\mathcal{G}_{(G)}=0$ gives a quadratic equation with respect to $a^{2}$ and $\nu^{2}$, we must require $g_{8} \geq 0$. Otherwise, tensor perturbations with large $\nu^{2}$ show unstable behavior.

To illustrate the stability of each perturbation mode, we first analyze the lowest order of the tensor perturbation in a closed FLRW background, namely, the $K=1$ and $n=3$ $\left(\nu^{2}=8\right)$ case. Then, we find

$$
\begin{aligned}
\mathcal{G}_{(G)}= & \frac{8}{a^{2}}+\frac{16}{3 a^{4}}\left(12 g_{3}-g_{r}\right) \\
& +\frac{8}{a^{6}}\left[48\left(g_{56}+g_{8}\right)-g_{s}\right] .
\end{aligned}
$$

In order to stabilize this perturbation mode for any $a>0$, the following condition must be satisfied:

$$
\begin{gathered}
-\frac{1}{9}\left(g_{r}-12 g_{3}\right)^{2} \geq g_{s}-48\left(g_{56}+g_{8}\right) \quad \text { for } g_{r} \geq 12 g_{3}, \\
0 \geq g_{s}-48\left(g_{56}+g_{8}\right) \quad \text { for } g_{r}<12 g_{3} .
\end{gathered}
$$

One may notice that the stability condition (4.9) reproduces the result shown in Ref. [15]. In fact, the tensor perturbations with $n=3$ in a closed FLRW background include homogeneous and anisotropic perturbation, namely, 
TABLE I. The conditions and properties of bouncing solutions with a positive cosmologiacal constant. The top table (a) is the case of closed FLRW and the bottom table (b) is the case of open FLRW. N/A means that there is no corresponding value of the scale factor.

(a) closed FLRW $(K=1)$

Solutions

Conditions

Domain

$\mathcal{B}_{B C}^{[1]}(\mathrm{i})$

$0<\tilde{g}_{s}<\tilde{g}_{s}^{[1](+)}$

$\tilde{a} \leq \tilde{a}_{2}^{[1]}, \tilde{a} \geq \tilde{a}_{3}^{[1]}$

$a_{B C}$

$a_{B C} \quad a_{\text {mim }}$

N/A

$\tilde{a}_{3}^{[1]}$

$\mathcal{B}_{B C}^{[1]}(\mathrm{ii})$

$\tilde{g}_{s}=0,0<\tilde{g}_{r}<\frac{3}{4}$

$\mathcal{B}^{[1]}(\mathrm{i})$

$$
\tilde{g}_{s}^{[1](+)}<\tilde{g}_{s}<0, \tilde{g}_{r}<1
$$

$\tilde{a} \leq \tilde{a}_{-}^{[1]}, \tilde{a} \geq \tilde{a}_{+}^{[1]}$

$\tilde{a}_{-}^{[1]}$

N/A

N/A

$\tilde{a}_{+}^{[1]}$

$\mathcal{B}^{[1]}($ ii $)$

$$
\begin{cases}\tilde{g}_{s}<0, \tilde{g}_{s}<\tilde{g}_{s}^{[1](-)} & \text { for }\left|2 \tilde{g}_{r}-1\right|<1 \\ \tilde{g}_{s}<0 & \text { for }\left|2 \tilde{g}_{r}-1\right| \geq 1\end{cases}
$$

$\tilde{a} \geq \tilde{a}_{1}^{[1]}$

N/A

N/A

N/A

$\tilde{a}_{1}^{[1]}$

$\mathcal{B}^{[1]}($ iii $)$

$\tilde{g}_{s}=0, \tilde{g}_{r} \leq 0$

$\tilde{a} \geq \tilde{a}_{3}^{[1]}$

N/A

N/A

N/A

$\tilde{a}_{3}^{[1]}$

$\mathcal{B}_{\mathcal{O}}^{[1]}$

$\tilde{g}_{s}<0, \tilde{g}_{s}^{[1](-)} \leq \tilde{g}_{s}<\tilde{g}_{s}^{[1](+)}, 0<\tilde{g}_{r}<1$

$\tilde{a} \geq \tilde{a}_{+}^{[1]}$
$\tilde{a}_{1}^{[1]} \leq \tilde{a} \leq \tilde{a}_{2}^{[1]}, \tilde{a} \geq \tilde{a}_{3}^{[1]}$

N/A

N/A

$\begin{array}{lll}\text { N/A } & \text { N/A } & \tilde{a}_{+}^{[1]} \\ \tilde{a}_{1}^{[1]} & \tilde{a}_{2}^{[1]} & \tilde{a}_{3}^{[1]}\end{array}$

(b) open FLRW $(K=-1)$

\begin{tabular}{lccccc} 
Solutions & Conditions & Domain & $a_{B C}$ & $a_{\operatorname{mim}}$ & $a_{\max }$ \\
\hline $\mathcal{B}_{B C}^{[-1]}$ & $0 \leq \tilde{g}_{s}<\tilde{g}_{s}^{[-1](+)}, \tilde{g}_{r}<0$ & $\tilde{a} \leq \tilde{a}_{2}^{[-1]}, \tilde{a} \geq \tilde{a}_{3}^{[-1]}$ & $\tilde{a}_{2}^{[-1]}$ & N/A & N/A \\
$\mathcal{B}^{[-1]}(\mathrm{i})$ & $\tilde{g}_{s}<0$ & $\tilde{a} \geq \tilde{a}_{3}^{[-1]}$ & N/A & N/A & N/A \\
$\mathcal{B}^{[-1]}(\mathrm{ii})$ & $\tilde{g}_{s}=0, \tilde{g}_{r}<0$ & $\tilde{a} \geq \tilde{a}_{+}^{[-1]}$ & N/A & N/A & $\tilde{a}_{3}^{[-1]}$ \\
\hline \hline
\end{tabular}
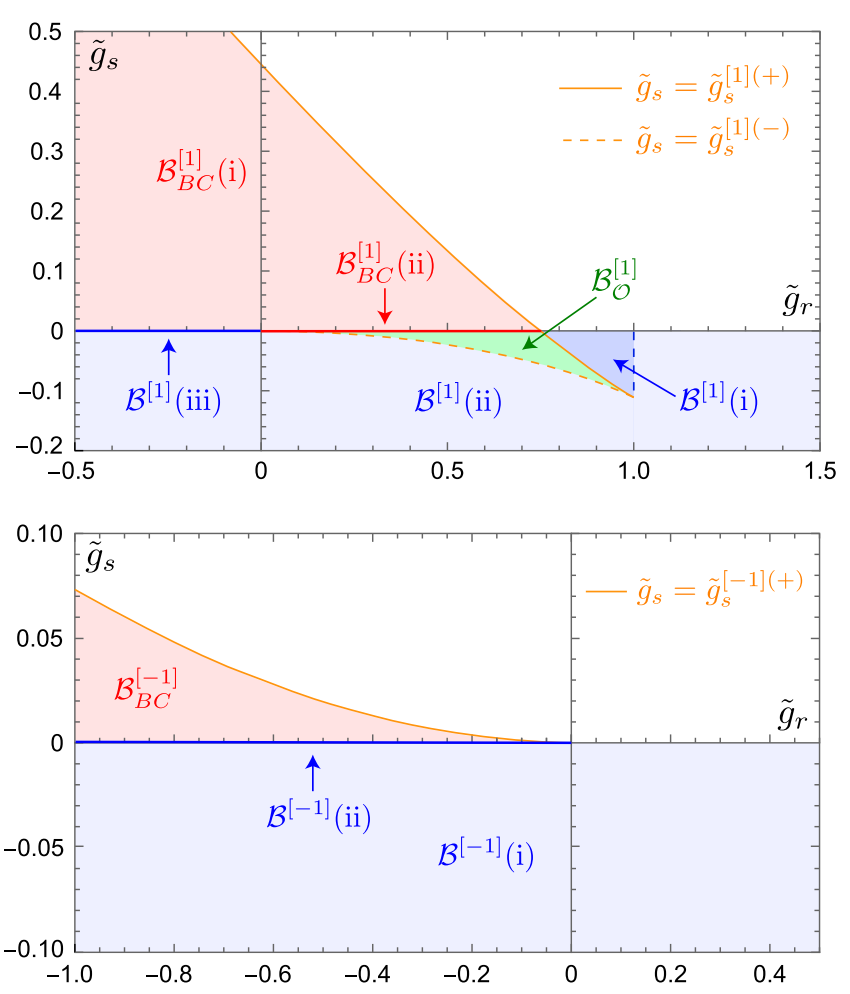

FIG. 3. The distribution of the bouncing solutions in the $\left(\tilde{g}_{r}, \tilde{g}_{s}\right)$ plane. The top and bottom figures correspond to the case with $K=1$ and $K=-1$, respectively. The red, blue, and green regions indicate the solutions of $\mathcal{B}_{B C}^{[K]}, \mathcal{B}^{[K]}$, and $\mathcal{B}_{\mathcal{O}}^{[1]}$, respectively. In particular, the solutions with $\tilde{g}_{s}=0$, namely, $\mathcal{B}_{B C}^{[1]}(\mathrm{ii}), \mathcal{B}^{[1]}$ (iii), and $\mathcal{B}^{[-1]}(\mathrm{ii})$, are indicated by colored lines.
Bianchi type-IX spacetime with a small anisotropy (see Sec. IV D).

It is obvious that we should impose the positivity of $\mathcal{G}_{(G)}$ for any perturbation mode $\nu^{2}$ to ensure the stability of spacetime against a tensor perturbation. Of course, we can express the stability conditions for a tensor perturbation for any viable $a>0$ and perturbation mode $\nu^{2}$ in terms of the coupling constant $g_{i}$. Since $\mathcal{G}_{(G)}=0$ gives a quadratic equation in terms of $a^{2}$ and $\nu^{2}$, we can solve it, in principle. However, it is found that the explicit form is quite complicated. In particular, $\nu^{2}$ takes a discrete value in a closed background, and this fact complicates the analysis. Thus, instead of considering a general case, we show a special case, namely, $g_{3}=0$ in open FLRW spacetime. Then, we find that every mode of the tensor perturbation can be stabilized for any $a \geq 0$, if one of the following four conditions is satisfied:

(i) $0<g_{8} \leq g_{56}, \quad g_{r}>0, \quad g_{s} \geq \frac{\left(g_{8}-3 g_{56}\right)^{2}}{g_{8}}$;

(ii) $0<g_{8} \leq g_{56}, \quad g_{r} \leq 0, \quad g_{s} \geq \frac{\left(g_{8}-3 g_{56}\right)^{2}}{g_{8}}+\frac{g_{r}^{2}}{9}$;

(iii) $g_{56}<g_{8}, \quad g_{r}>0, \quad g_{s} \geq 4\left(3 g_{56}-2 g_{8}\right)$;

(iv) $g_{56}<g_{8}, \quad g_{r} \leq 0, \quad g_{s} \geq 4\left(3 g_{56}-2 g_{8}\right)+\frac{g_{r}^{2}}{9}$.

Note that there is some difficulty in reconciling the above conditions with the conditions for bouncing solutions in open FLRW spacetime. Referring to Table I and Fig. 3, one 
can see that $\mathcal{B}_{B C}^{[-1]}$ appears if $g_{r}<0$ and $g_{s}>0$ with $\left|\tilde{g}_{r}\right| \gg\left|\tilde{g}_{s}\right|$. On the other hand, $\mathcal{B}^{[-1]}$ can be found if $g_{s}<0$. Then, we shall examine compatibilities of these conditions.

(i) Condition (i) is incompatible with both types of bouncing solutions, because $g_{r}$ and $g_{s}$ are constrained to be positive, which completely contradicts both conditions for $\mathcal{B}_{B C}^{[-1]}$ and $\mathcal{B}^{[-1]}$.

(ii) Since $g_{s}$ is constrained to be a positive number under condition (ii), there is no stable bouncing solution $\mathcal{B}^{[-1]}$. Additionally, one may notice that the lower bound of $g_{s}$ is given in terms of $g_{r}^{2}$. Since $\left|\tilde{g}_{r}\right| \gg\left|\tilde{g}_{s}\right|$ should be satisfied to stabilize the solution $\mathcal{B}_{B C}^{[-1]}$, condition (ii) is hard to compatible unless small $\Lambda>0$ is set. As we show later, small $\Lambda>0$ is not preferable to stabilize the scalar perturbation at the infrared regime.

(iii) Since $g_{r}$ is constrained to be positive, condition (iii) cannot be compatible with the solution $\mathcal{B}_{B C}^{[-1]}$. The bouncing solution $\mathcal{B}^{[-1]}$ can be stabilized only if $3 g_{56}>2 g_{8}$.

(iv) Under condition (iv), both $\mathcal{B}_{B C}^{[-1]}$ and $\mathcal{B}^{[-1]}$ can be stabilized. Since $g_{s}$ is bounded below by $g_{r}^{2}$, it may require a certain level of tuning to stabilize the bouncing solution $\mathcal{B}_{B C}^{[-1]}$ for the same reason as condition (ii).

In the general case, we also anticipate unstable tensor perturbations in bouncing open FLRW spacetime. From (3.25), one can see that $g_{s}>0$ and $g_{r}>0$ are preferable in order to ensure the positivity of $\mathcal{G}_{(G)}$, which are basically contradicting to the conditions of bouncing solutions $\mathcal{B}_{B C}^{[-1]}$ and $\mathcal{B}^{[-1]}$. Conversely, in closed FLRW spacetime, the tensor perturbations can be stabilized without special tuning. Naively, $g_{r}<0$ and $g_{s}<0$ are preferred to satisfy the positivity of $\mathcal{G}_{(G)}$ for any $a>0$ and $\nu^{2}$. Referring to Table I and Fig. 3, we find the $\mathcal{B}^{[1]}$ (ii) solution is under such a condition.

\section{Scalar perturbation}

Although the negative sign of $\mathcal{G}_{(Q)}$ in the infrared regime cannot be avoided, $\mathcal{G}_{(Q)}$ may take a positive value in the deep ultraviolet region in which the effects of higher spatial curvatures are predominant. To realize a stable bouncing phase, we require the positivity of $\mathcal{G}_{(Q)}$, at least in the range $\left[0, a_{\text {ini }}\right]$ with $a_{\text {ini }}>a_{T}$. Since $\mathcal{G}_{(Q)}=0$ gives a quadratic equation in terms of $a^{2}$ with an upward convex, the positivity of $\mathcal{G}_{(Q)}$ at least in $\left[0, a_{\mathrm{ini}}\right]$ is guaranteed if both of the following conditions are satisfied:

$$
\begin{aligned}
0 \leq & -9 a_{\mathrm{ini}}^{4}+\left[\frac{2 g_{r}}{K^{2}}\left(2 \nu^{2}-3 K\right)+3 g_{3} \nu^{2}\right] a_{\mathrm{ini}}^{2} \\
& +3\left[\frac{g_{s}}{K^{2}}\left(4 \nu^{2}-9 K\right)+2\left(3 g_{56}-4 g_{7}\right) K \nu^{2}\right. \\
& \left.+\left(-8 g_{7}+3 g_{8}\right) \nu^{2}\left(3 \nu^{2}-10 K\right)\right] \\
0 \leq & \frac{g_{s}}{K^{2}}\left(4 \nu^{2}-9 K\right)+2\left(3 g_{56}-4 g_{7}\right) K \nu^{2} \\
& +\left(-8 g_{7}+3 g_{8}\right) \nu^{2}\left(3 \nu^{2}-10 K\right)
\end{aligned}
$$

Note that the case with $g_{8}<8 g_{7} / 3$ must be excluded; otherwise, the scalar perturbations with large $\nu^{2}$ show unstable behavior.

In our analysis, we can find bouncing solutions $\mathcal{B}_{B C}^{[K]}$, $\mathcal{B}^{[K]}$, and $\mathcal{B}_{\mathcal{O}}^{[1]}$ which satisfy both the tensor and scalar stability conditions, namely, with $\mathcal{G}_{(G)} \geq 0$ in $[0, \infty)$ and $\mathcal{G}_{(Q)} \geq 0$ at least in $\left[0, a_{T}\right]$. The examples of such solutions are listed in Table II. Note that a certain level of tuning is required to find a stable open bouncing universe, because the tensor perturbation tends to be unstable for the reason we mentioned.

One may wonder about the appropriate value of $a_{\text {ini }}$. The initial scale factor $a_{\text {ini }}$ seems to relate with a quantum creation of the Universe. Therefore, it is natural to consider that the typical energy scale is estimated at the Planck scale. On the other hand, the typical scale of the bouncing radius is also expected to be around the Planck scale, because the bouncing behavior is induced by higher spatial curvature terms, namely, quantum gravitational corrections. More specifically, we assume

\begin{tabular}{|c|c|c|c|c|c|c|c|c|c|c|c|c|c|c|}
\hline & Type & $\Lambda$ & $g_{2}$ & $g_{3}$ & $g_{4}$ & $g_{5}$ & $g_{6}$ & $g_{7}$ & $g_{8}$ & $g_{r}$ & $g_{s}$ & $a_{T}$ & $a_{\text {crit }}$ & $a_{\text {crit }} / a_{T}$ \\
\hline (i) & $\mathcal{B}_{B C}^{[1]}$ & $\frac{3}{2}$ & -1 & 1 & $\frac{1}{5}$ & $-\frac{1}{2}$ & 1 & -1 & 1 & -12 & $\frac{78}{5}$ & 1.856 & 3.544 & 1.909 \\
\hline (ii) & $\mathcal{B}^{[1]}$ & 1 & $-\frac{29}{90}$ & 1 & $-\frac{7}{108}$ & $\frac{1}{2}$ & -1 & -1 & 1 & $\frac{1}{5}$ & -1 & 1.744 & 4.699 & 2.694 \\
\hline (iii) & $\mathcal{B}_{\mathcal{O}}^{[1]}$ & $\frac{1}{5}$ & $\frac{1}{5}$ & 1 & 0 & $-\frac{1}{5}$ & $\frac{1}{4}$ & $\frac{1}{4}$ & 1 & $\frac{48}{5}$ & $-\frac{21}{5}$ & 3.270 & 5.514 & 1.686 \\
\hline (iv) & $\mathcal{B}_{B C}^{[-1]}$ & $\frac{3}{2}$ & $-\frac{5}{18}$ & $\frac{2}{3}$ & $-\frac{133}{2160}$ & $\frac{1}{4}$ & $-\frac{1}{5}$ & $\frac{1}{16}$ & $\frac{1}{3}$ & -1 & $\frac{1}{20}$ & 0.485 & 1.380 & 2.849 \\
\hline (v) & $\mathcal{B}^{[-1]}$ & 1 & $\frac{1}{18}$ & $-\frac{2}{15}$ & $\frac{31}{108}$ & $-\frac{1}{2}$ & -1 & $\frac{1}{900}$ & $\frac{2}{225}$ & $\frac{1}{5}$ & -1 & 0.712 & 0.920 & 1.293 \\
\hline
\end{tabular}
the three-Ricci curvature represents the energy scale, namely, $m \sim \sqrt{\mathcal{R}} \propto a^{-1}$. Then, the ratio of quantum creation scale $m_{\text {ini }}$ to bouncing scale $m_{T}$ is given by

TABLE II. The examples for stable bouncing solutions with positive cosmological constants. 


$$
\frac{m_{\text {ini }}}{m_{T}} \sim \frac{a_{T}}{a_{\text {ini }}} .
$$

It is natural to consider the ratio is of the order of one.

Additionally, we consider the upper limit of $a_{\text {ini }}$ to satisfy the positivity of $\mathcal{G}_{(Q)}$ during the bouncing phase. As we noted, $\mathcal{G}_{(Q)}$ must be negative for large $a$. Thus, there exists a critical value of the scale factor $a_{\text {crit }}$. Namely, all scalar perturbation modes show $\mathcal{G}_{(Q)}>0$ for $a<a_{\text {crit }}$; however, any one of them turns to be zero at $a=a_{\text {crit }}$. Obviously, $a_{\text {ini }}$ must be in $\left(a_{T}, a_{\text {crit }}\right)$. A further constraint for $a_{\text {ini }}$ can be imposed by considering the dynamics of the perturbations.

\section{Dynamics of perturbations}

To construct a scenario for nonsingular cosmological evolution, we have to pay attention to the dynamics of the perturbations. Taking a variation of the quadratic actions with respect to $h_{(G)}$ and $h_{(Q)}$, we obtain the equations of motion for the tensor and scalar perturbations, respectively:

$$
\begin{aligned}
& \ddot{h}_{(G)}+3 H \dot{h}_{(G)}+\mathcal{M}_{(G)}^{2} h_{(G)}=0, \\
& \ddot{h}_{(Q)}+3 H \dot{h}_{(Q)}+\mathcal{M}_{(Q)}^{2} h_{(Q)}=0,
\end{aligned}
$$

where we define the squared effective masses of the tensor and scalar perturbation as

$$
\mathcal{M}_{(G)}^{2}:=\frac{\mathcal{G}_{(G)}}{\mathcal{F}_{(G)}}, \quad \mathcal{M}_{(Q)}^{2}:=\frac{\mathcal{G}_{(Q)}}{\mathcal{F}_{(Q)}} .
$$

We first consider the contracting phase before the bounce. In this era, the perturbations feel a Hubble acceleration which is derived from the second terms in (4.13) and (4.14) because of negative Hubble parameter $H<0$. Since the Hubble acceleration enhances both perturbation modes, this effect should be suppressed by effective mass terms.

Intuitively, the magnitudes of the Hubble accelerations for tensor and scalar perturbation are given by $H^{2}$. Thus, to suppress the unstable behavior, we require

$$
\mathcal{M}_{(G)}^{2} \gtrsim H^{2}, \quad \mathcal{M}_{(Q)}^{2} \gtrsim H^{2}
$$

throughout the contracting phase. This condition gives a further constraint on the possible value of the initial scale factor. Namely, $a_{\text {ini }}$ should be in the range of $\left(a_{T}, a_{H}\right)$, where $a_{H}$ is a value of the scale factor in which any one of the squared effective mass of perturbation turns out to be $\mathcal{M}^{2}=H^{2}$. Namely, for $a<a_{H}$, every perturbation mode shows a positive squared effective mass which is larger than $H^{2}$. Then, the condition (4.16) is ensured in the contracting era for $a_{T}<a_{\text {ini }}<a_{H}$.

Note that the possible range for the initial scale factor can be broadened by tuning the value of coupling constant $\lambda$.
Namely, the dependence of $\lambda$ in effective mass-Hubble parameter ratios is naively evaluated as follows:

$$
\frac{\mathcal{M}_{(G)}^{2}}{H^{2}} \propto 3 \lambda-1, \quad \frac{\mathcal{M}_{(Q)}^{2}}{H^{2}} \propto \lambda-1 ;
$$

then one can see that a large value of $\lambda$ weakens the effect of Hubble acceleration in both cases.

After the bounce at $a_{T}$, the universe turns to expand. As we mentioned, the squared effective mass of the scalar perturbation must be negative in the infrared regime. Thus, to stabilize the perturbation, Hubble friction with $H>0$ must overcome the effects of the negative squared effective mass of scalar modes. Namely, we require

$$
\left|\mathcal{M}_{(Q)}^{2}\right| \lesssim H^{2} \quad \text { when } \mathcal{M}_{(Q)}^{2}<0
$$

in the expanding era with a large value of the scale factor.

We examine the stability of the bouncing solutions by showing concrete examples. First, we shall show the bouncing solution without any instability throughout the evolution. In Fig. 4, we show the evolutions of $\mathcal{M}_{(G)}^{2} / H^{2}$ and $\mathcal{M}_{(Q)}^{2} / H^{2}$ in terms of the scale factor. From these figures, we find that $\mathcal{M}_{(G)}^{2}$ of all tensor perturbation modes are always positive, and the condition (4.18) is always satisfied after turning $\mathcal{M}_{(Q)}^{2}$ to be negative for any scalar perturbation modes. Thus, the instabilities in the scalar perturbation are suppressed by Hubble friction, in this case.

On the other hand, we show an example of a bouncing solution with temporal scalar instabilities in Fig. 5. Although all of the tensor perturbations show stable behavior because of positive $\mathcal{M}_{(G)}^{2}$, some of the scalar perturbations temporally show tachyon instabilities. Namely, for a temporary period, some scalar perturbation modes violate the condition (4.18).

One may wonder about the growth of a scalar perturbation during tachyon instability. Seeing the equation of motion for a scalar perturbation, it is natural to speculate that the growth rate is related with the minimum value of $\mathcal{M}_{(Q)}^{2} / H^{2}$. Thus, we first clarify the minimum value of the squared effective mass $\mathcal{M}_{(Q)}^{2}$. For simplicity, we consider the asymptotic region, i.e., for large perturbation mode $\nu^{2}$. In this limit, the kinetic and mass terms of scalar perturbations given by (3.26) and (3.27) are reduced into the following forms:

$$
\mathcal{F}_{(Q)} \approx \frac{2(3 \lambda-1)}{3(\lambda-1)}
$$

$\mathcal{G}_{(Q)} \approx-\frac{2 \nu^{2}}{3 a^{2}}+\frac{2 \nu^{4}}{27 a^{4}}\left[\frac{4 g_{r}}{K^{2}}+3 g_{3}\right]+\frac{2 \nu^{6}}{3 a^{6}}\left(3 g_{8}-8 g_{7}\right)$. 

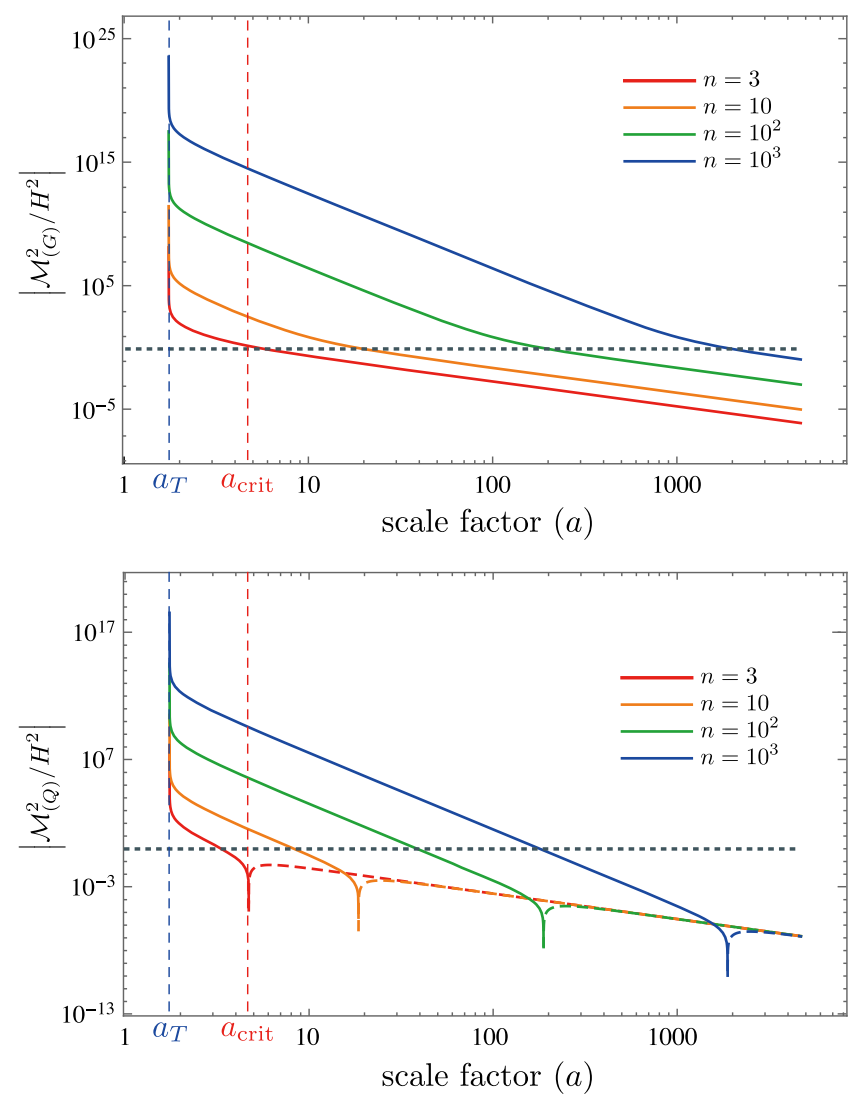

FIG. 4. The typical example of a bouncing solution without any instability after a bounce. In this figure, we show the evolutions of solution (ii) listed in Table II. We set the coupling constant $\lambda$ to be unity. The red, orange, green, and blue curves (from bottom to top) indicate the ratio of the squared effective masses to squared Hubble parameter with $n=3,10,10^{2}$, and $10^{3}\left(\nu^{2}=n^{2}-1\right)$, respectively. The top and bottom figures show those of the tensor and scalar perturbations, respectively. The solid (dashed) curve shows the evolution with $\mathcal{M}^{2}>0\left(\mathcal{M}^{2}<0\right)$. The gray dotted line indicates $\mathcal{M}^{2}=H^{2}$.

Then, the minimum value of $\mathcal{M}_{(Q)}^{2}$ is given by

$$
\begin{aligned}
\min _{a} \mathcal{M}_{(Q)}^{2} \approx & -\frac{3 K^{2}(\lambda-1)}{(3 \lambda-1) \eta^{2}}\left[2 \eta-\left(4 g_{r}+3 g_{3} K^{2}\right)\right] \quad \text { with } \\
\eta:= & 4 g_{r}+3 g_{3} K^{2} \\
& +\sqrt{243\left(3 g_{8}-8 g_{7}\right) K^{4}+\left(4 g_{r}+3 g_{3} K^{2}\right)^{2}} .
\end{aligned}
$$

After taking the above value, $\mathcal{M}_{(Q)}^{2}$ monotonically increases with time and approaches zero. Then, the scalar perturbation is stabilized by Hubble friction. The important point is that the minimum value does not depend on the perturbation mode $\nu^{2}$ in this limit. Thus, we can conclude that the minimum value of $\mathcal{M}_{(Q)}^{2}$ can be bounded in a finite value. Namely, if the ratio to the Hubble parameter for large $\nu^{2}$,
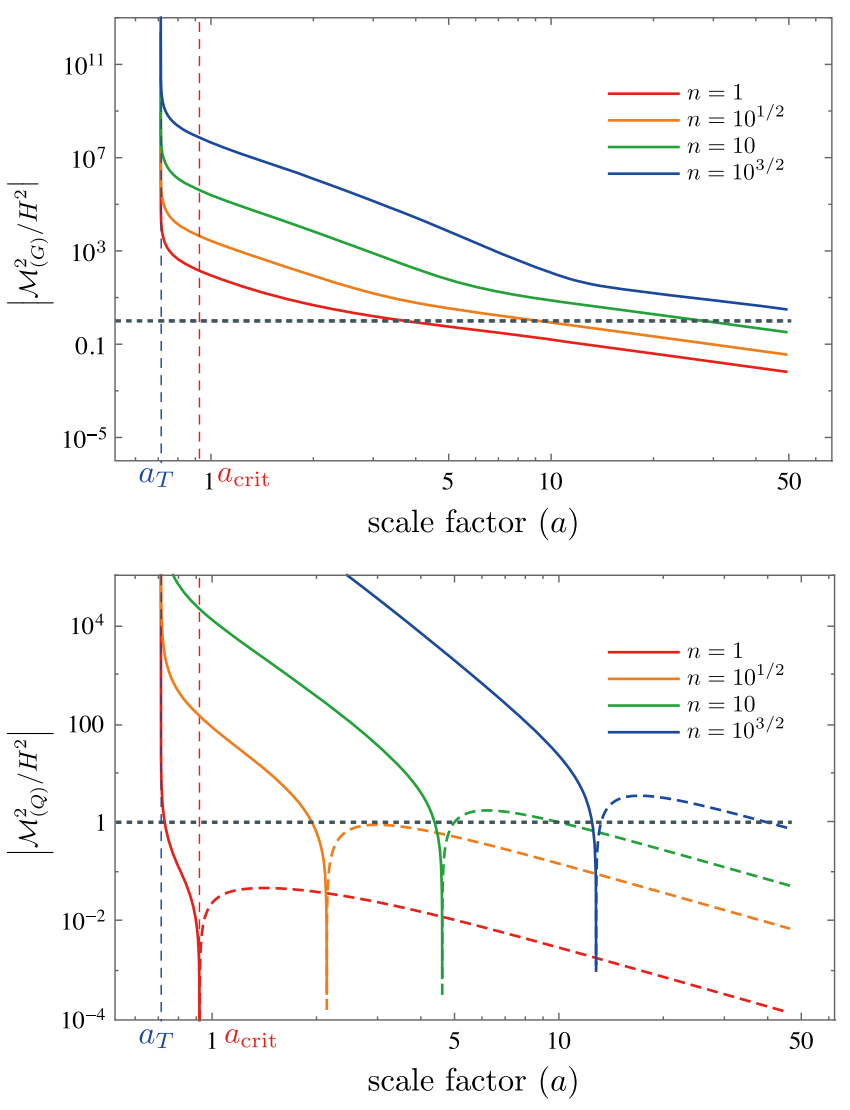

FIG. 5. The typical example of a bouncing solution with temporal scalar instabilities. In this figure, we show the evolutions of solution (v) listed in Table II. We set the coupling constant $\lambda=2.1$. The red, orange, green, and blue curves (from bottom to top) indicate the ratio of the squared effective masses to squared Hubble parameter with $n=1,10^{1 / 2}, 10$, and $10^{3 / 2}$ $\left(\nu^{2}=n^{2}+1\right)$, respectively. The top and bottom figures show those of the tensor and scalar perturbations, respectively. The solid (dashed) curve shows the evolution with $\mathcal{M}^{2}>0$ $\left(\mathcal{M}^{2}<0\right)$. The gray dotted line indicates $\mathcal{M}^{2}=H^{2}$.

$$
\min _{a} \frac{\mathcal{M}_{(Q)}^{2}}{H^{2}} \approx-\frac{9 K^{2}(\lambda-1)}{2 \Lambda \eta^{2}}\left[2 \eta-\left(4 g_{r}+3 g_{3} K^{2}\right)\right],
$$

is sufficiently suppressed, it is expected that there is no serious instability at least at the classical level. Note that the large value of the positive cosmological constant $\Lambda$ and/or the small value of $\lambda-1>0$ decreases the above value.

In the above discussion, we limited our analysis to the case with large $\nu^{2}$. However, we can investigate the case with an intermediate value of $\nu^{2}$ in the same manner, and we find the minimum value of $\mathcal{M}_{(Q)}^{2} / H^{2}$ is also affected by the values of $\Lambda$ and $\lambda$. Namely, large $\Lambda>0$ and small $\lambda-1>0$ are preferred.

Then, we demonstrate the growth of the scalar perturbation without condition (4.18) by solving the equation of motion, numerically. In Fig. 6, we show the evolution of the 


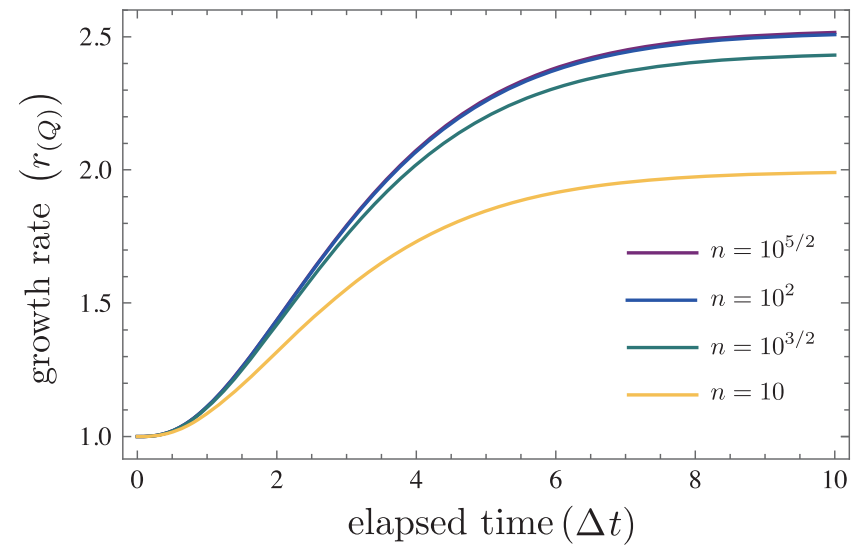

FIG. 6. The evolutions of the scalar perturbation growth rates $r_{(Q)}:=h_{(Q)}(t) / h_{(Q)}\left(t_{0}\right)$ in terms of $n$ [solution (v) listed in Table II]. In this numerical simulation, we set $\dot{h}_{(Q)}\left(t_{0}\right)=0$ and $\lambda=2.1$. The yellow, green, blue, and violet curves (from bottom to top) indicate the growth rate with $n=10,10^{3 / 2}, 10^{2}$, and $10^{5 / 2}$, respectively. Note that the purple and blue curves are almost degenerated. The elapsed time is defined by $\Delta t:=t-t_{0}$. In this solution, $\min _{a}\left(\mathcal{M}_{(Q)}^{2} / H^{2}\right)$ approaches -3.621 for a large perturbation mode.

scalar perturbation of solution (v) listed in Table II. In this case, $\min _{a}\left(\mathcal{M}_{(Q)}^{2} / H^{2}\right)$ monotonically decreases as the perturbation mode becomes larger and approaches -3.621 (see Fig. 5). Then, the growth rate of the scalar perturbation is converged to $r_{(Q)}(\infty):=h_{(Q)}(\infty) /$ $h_{(Q)}\left(t_{0}\right) \approx 2.527$ for a large perturbation mode, where $t_{0}$ is a time at which $\mathcal{M}_{(Q)}^{2}=0$. Since the growth rate can be suppressed as $r_{(Q)} \sim \mathcal{O}(1)$, the temporal tachyon instability may not provide a serious effect to the background geometry.

Furthermore, we mention the relation between the scalar growth rate and the minimum value (4.22). To evaluate the relation, we perform a numerical calculation by setting various $\lambda$ with a fixed perturbation mode. Then, the evolution of the scalar perturbation is shown in Fig. 7. Additionally, the detailed data of the asymptotic values of $r_{(Q)}$ in terms of $\lambda$ are shown in Table III. From this result, we can conclude that the large value of $\left|\mathcal{M}_{(Q)}^{2} / H^{2}\right|$ with $\mathcal{M}_{(Q)}^{2}<0$ enhances the growth of the scalar perturbation. In other words, the scalar growth rate is amplified by choosing a large value of $\lambda$ and a small value of $\Lambda>0$.

When the accelerating expansion caused by a cosmological constant persists, the effect of the spatial curvature $K$ turns out to be irrelevant. Then, the analysis can be simplified into the case with $K=0$ and $\Lambda>0$. In other words, the spacetime can be approximated as the de Sitter solution at the late time of the evolution after the bounce (cf. the cosmic no-hair theorem [28]). It should be noted that the detailed analysis of de Sitter spacetime stability has been already performed in Ref. [19]. In those papers, the

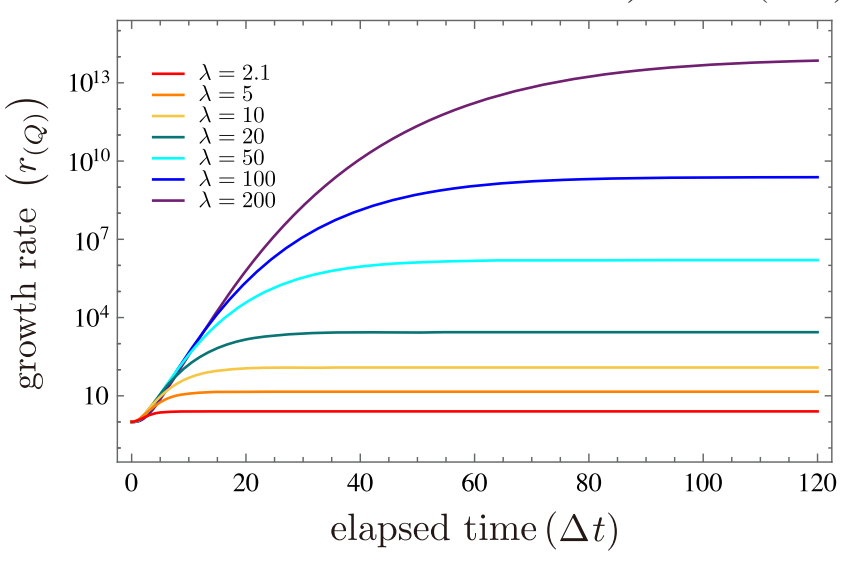

FIG. 7. The evolutions of the scalar perturbation growth rates $r_{(Q)}:=h_{(Q)}(t) / h_{(Q)}\left(t_{0}\right)$ in terms of $\lambda$ [solution (v) listed in Table II]. In this plot, we set $\dot{h}_{(Q)}\left(t_{0}\right)=0$ and $n=10^{5 / 2}$. The red, orange, yellow, green, cyan, blue, and violet curves (from bottom to top) indicate the growth rate with $\lambda=2.1,5,10,20$, 50,100 , and 200, respectively. The elapsed time is defined by $\Delta t:=t-t_{0}$.

authors indicated that the instability of the scalar perturbation may be cured if the background spacetime is the de Sitter solution. It is worth mentioning that the quadratic actions in flat FLRW spacetime can be reproduced by simply taking a limit $K \rightarrow 0$ in (3.22) and (3.23).

\section{Backreaction of the perturbation on background geometry}

We further discuss the stability of a bouncing solution by considering a backreaction of the perturbation, in particular, against anisotropic and homogeneous perturbation in closed FLRW spacetime. Such perturbation modes can be derived by considering Bianchi type-IX spacetime whose three-dimensional space is homogeneous; however, isotropy does not always hold. The metric is given by

$$
d s^{2}=-d t^{2}+\frac{a^{2}}{4} e^{2 \beta_{i j}} \omega^{i} \omega^{j},
$$

TABLE III. The detailed data of the relation between the value of $\lambda$ and the asymptotic value of the scalar growth rate. In this table, we set $n=10^{5 / 2}$ [solution (v) listed in Table II].

\begin{tabular}{lcc}
\hline \hline$\lambda$ & $\min \left(\mathcal{M}_{(Q)}^{2} / H^{2}\right)$ & $r_{(Q)}$ \\
\hline 2.1 & -3.621 & 2.527 \\
5 & $-1.317 \times 10^{1}$ & $1.437 \times 10^{1}$ \\
10 & $-2.963 \times 10^{1}$ & $1.218 \times 10^{2}$ \\
20 & $-6.255 \times 10^{1}$ & $2.749 \times 10^{3}$ \\
50 & $-1.613 \times 10^{2}$ & $1.596 \times 10^{6}$ \\
100 & $-3.259 \times 10^{2}$ & $2.418 \times 10^{9}$ \\
200 & $-6.551 \times 10^{2}$ & $8.747 \times 10^{13}$ \\
\hline \hline
\end{tabular}


where $a$ represents the scale factor and $\omega^{i}(i=1,2,3)$ is an invariant basis, which is given by

$$
\begin{aligned}
& \omega^{1}=\sin x^{3} \sin x^{2} d x^{1}+\cos x^{3} d x^{2}, \\
& \omega^{2}=-\cos x^{3} \sin x^{2} d x^{1}+\sin x^{3} d x^{2}, \\
& \omega^{3}=\cos x^{2} d x^{1}+d x^{3} .
\end{aligned}
$$

The traceless symmetric tensor $\beta_{i j}$ represents anisotropy. Since Bianchi type-IX space belongs to Bianchi class $\mathrm{A}, \beta_{i j}$ can be diagonalized without loss of generality as follows [29]:

$$
\beta_{i j}=\operatorname{diag}\left(\beta_{+}+\sqrt{3} \beta_{-}, \beta_{+}-\sqrt{3} \beta_{-},-2 \beta_{+}\right) .
$$

When $\beta_{ \pm}=0$, the spatial isotopy is restored, namely, closed FLRW spacetime. The basic equations are given by

$$
\begin{gathered}
\dot{H}+3 H^{2}-\frac{8}{3(3 \lambda-1)}\left[\frac{8}{a^{5}} \frac{\partial V}{\partial a}+\frac{3 \mathcal{C}_{\mathrm{IX}}}{a^{3}}\right]=0, \\
\ddot{\beta}_{ \pm}+3 H \dot{\beta}_{ \pm}+\frac{32}{3 a^{6}} \frac{\partial V}{\partial \beta_{ \pm}}=0, \\
H^{2}=\frac{2}{3(3 \lambda-1)}\left[3\left(\dot{\beta}_{+}^{2}+\dot{\beta}_{-}^{2}\right)+\frac{64}{a^{6}} V\left(a, \beta_{ \pm}\right)+\frac{8 \mathcal{C}_{\mathrm{IX}}}{a^{3}}\right],
\end{gathered}
$$

where $V\left(a, \beta_{ \pm}\right):=-a^{3} \mathcal{L}_{P} / 128$ is a potential which is given by the spatial Ricci curvature terms (in Ref. [15], the explicit form is shown). Equations (4.26) and (4.27) correspond to the dynamical equations of the scale factor $a$ and the anisotropy $\beta_{ \pm}$, respectively. As is the case with the case of FLRW spacetime, we obtain a Friedmann-like equation (4.28). Because of integration with respect to the time variable, an integration constant $\mathcal{C}_{\mathrm{IX}}$ appears. For simplicity, we consider the case with $\mathcal{C}_{\mathrm{IX}}=0$.

Assuming $\left|\beta_{ \pm}\right| \ll 1$, the potential $V$ is reduced into

$$
V\left(a, \beta_{ \pm}\right) \approx U_{0}(a)+U_{2}(a)\left(\beta_{+}^{2}+\beta_{-}^{2}\right),
$$

where $U_{0}(a)$ and $U_{2}(a)$ are defined, respectively, by

$$
\begin{aligned}
U_{0}(a):=- & \frac{3 a^{4}}{64}\left[1-\frac{\Lambda}{3} a^{2}-\frac{g_{r}}{3 a^{2}}-\frac{g_{s}}{3 a^{4}}\right], \\
U_{2}(a):= & \frac{3 a^{6}}{64}\left[\frac{8}{a^{2}}+\frac{16}{3 a^{4}}\left(12 g_{3}-g_{r}\right)\right. \\
& \left.+\frac{8}{a^{6}}\left\{48\left(g_{56}+g_{8}\right)-g_{s}\right\}\right] .
\end{aligned}
$$

It is worth mentioning that $U_{0}$ and $U_{2}$ are related to the potential in FLRW spacetime (4.2) and the squared effective mass $\mathcal{M}^{2}:=\mathcal{G} / \mathcal{F}$ of the $n=3$ tensor mode as follows:

$$
\begin{gathered}
\mathcal{U}(a)=-\frac{64}{3(3 \lambda-1) a^{4}} U_{0}(a), \\
\left.\mathcal{M}_{(G)}^{2}\right|_{n=3}=\frac{64}{3 a^{6}} U_{2}(a) .
\end{gathered}
$$

One may notice that the equation of motion for the tensor perturbation with $n=3$ is reproduced if $\beta_{ \pm}$is replaced into $h_{(G)}$ in (4.27) [see (4.8) and (4.13)]. In other words, these perturbation modes include the homogeneous and anisotropic perturbation in closed FLRW spacetime. Then, Eq. (4.28) can be rewritten as follows:

$\frac{1}{2} \dot{a}^{2}+\mathcal{U}(a) \approx \frac{2 a^{2}}{3 \lambda-1}\left[E_{\beta_{+}}\left(a, \beta_{+}\right)+E_{\beta_{-}}\left(a, \beta_{-}\right)\right]$,

where

$$
E_{\beta_{ \pm}}\left(a, \beta_{ \pm}\right):=\frac{1}{2}\left[\dot{\beta}_{ \pm}^{2}+\left.\mathcal{M}_{(G)}^{2}\right|_{n=3} \beta_{ \pm}^{2}\right] .
$$

Since we impose the positivity of the tensor squared effective mass $\mathcal{M}_{(G)}^{2}$ to stabilize the perturbation, $E_{\beta_{ \pm}}$ always takes a positive value. From (4.34), one can see that the scale factor is regarded as a particle with energy $2 a^{2}\left(E_{\beta_{+}}+E_{\beta_{-}}\right) /(3 \lambda-1)$ in potential $\mathcal{U}(a)$. Namely, the possible range for the scale factor is broadened due to an anisotropic energy $E_{\beta_{ \pm}}$.

Then, we examine the backreaction on the singularityfree solutions, especially $\mathcal{B}_{B C}^{[1]}$. This type of bouncing solution realizes singularity avoidance due to the potential barrier $\mathcal{U} \geq 0$ between $a_{B C}$ and $a_{T}$ (see Fig. 1). However, the cosmological bounce at $a_{T}$ may be spoiled if the backreaction from an anisotropic perturbation is considered. Namely, the energy for the scale factor is lifted up to $2 a^{2}\left(E_{\beta_{+}}+E_{\beta_{-}}\right) /(3 \lambda-1)$ due to the anisotropic effect, and then the potential barrier can be overleaped if the anisotropic energy exceeds the local maximum value of the potential $\mathcal{U}$. In Fig. 8, we show the typical example based on solution (i) listed in Table II. In this analysis, we set $\lambda=1$, and the initial conditions are given by $a_{\mathrm{ini}}=1.5 a_{T} \approx 2.784, \beta_{ \pm}=\beta_{\text {ini }}$, and $\dot{\beta}_{ \pm}=0$. In the top figure, the evolutions of the anisotropic energies are shown. The anisotropic energy $2 a^{2}\left(E_{\beta_{+}}+E_{\beta_{-}}\right) /(3 \lambda-1)$ takes a maximum value at the bouncing time $t=t_{T} \approx 1.261$. If the initial anisotropy exceeds a critical value $\beta_{\text {crit }} \approx 0.0569$, the universe results in a big crunch by overleaping the potential barrier (the purple curves in the top figure of Fig. 8).

We further mention the dynamics of the anisotropy $\beta_{ \pm}$ and $\dot{\beta}_{ \pm}$(the bottom figure of Fig. 8). One may notice that the oscillating amplitudes of $\beta_{ \pm}$are almost invariant throughout the bounce, while those of $\dot{\beta}_{ \pm}$are enhanced whose maximum amplitudes reach up to $10^{-1}$ order. It is 

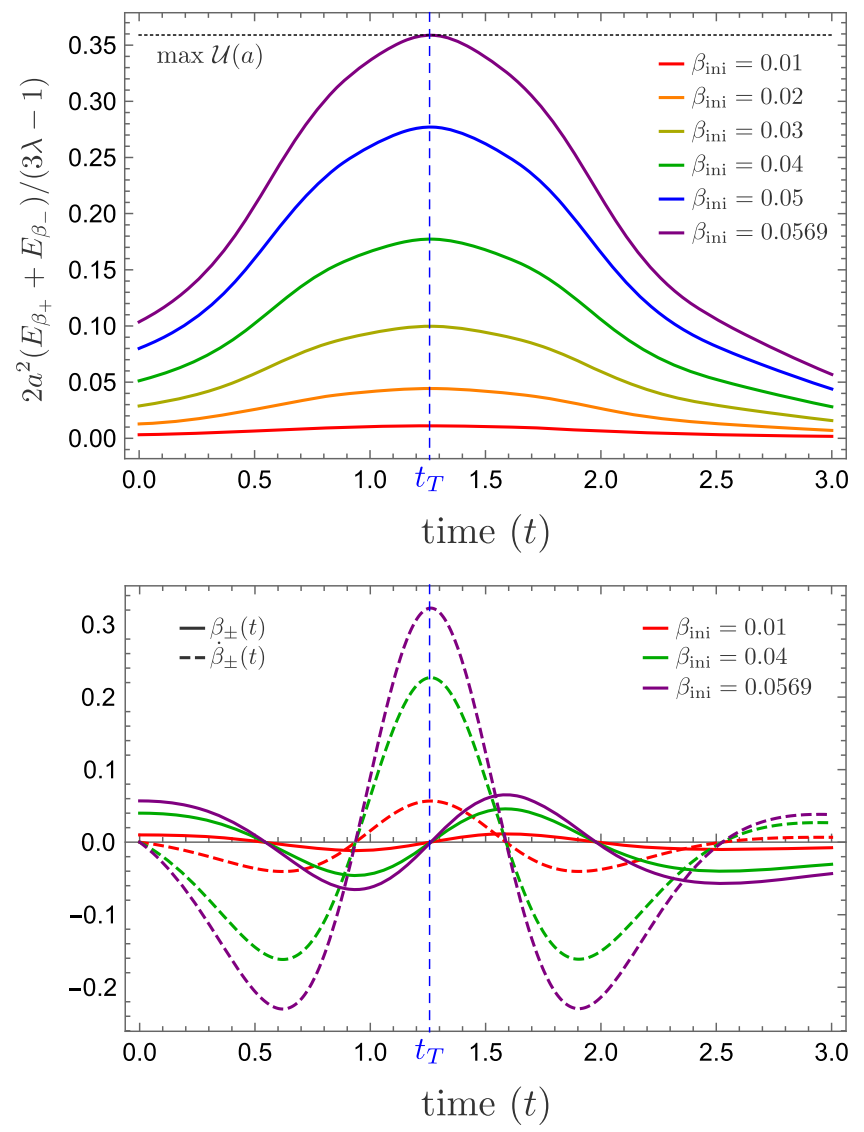

FIG. 8. The evolutions of anisotropy of $\mathcal{B}_{B C}^{[1]}$ [solution (i) listed in Table II]. In the top figure, the evolutions of anisotropic energies are shown. The initial conditions are given by $a_{\text {ini }}=1.5 a_{T} \approx 2.784, \beta_{ \pm}=\beta_{\text {ini }}$, and $\dot{\beta}_{ \pm}=0$. The coupling constant $\lambda$ is set to be unity. The red, orange, yellow, green, blue, and purple curves (from bottom to top) correspond to the anisotropic energy with initial anisotropy $\beta_{\text {ini }}=0.01,0.02,0.03,0.04,0.05$, and 0.0569 , respectively. The maximum value of the potential in FLRW spacetime $\mathcal{U} \approx 0.359$ is denoted by the gray dotted line. In the bottom figure, the solid and dashed curves indicate the evolutions of $\beta_{ \pm}$and $\dot{\beta}_{ \pm}$, respectively. The red, green, and purple curves correspond to the evolutions with initial condition $\beta_{\text {ini }}=0.01,0.04$, and 0.0569 , respectively.

not quite unnatural, because the dynamics of the anisotropic perturbation is approximately governed by the following equation:

$$
\ddot{\beta}_{ \pm} \approx-\mathcal{M}_{(G)}^{2} \beta_{ \pm}
$$

Around the bouncing point, the term including the Hubble parameter can be ignored. Then, the oscillating frequency is naively given by $\omega \approx \mathcal{M}_{(G)}=\sqrt{\mathcal{G}_{(G)} / \mathcal{F}_{(G)}}$. As a result, the amplitudes of $\dot{\beta}_{ \pm}$are approximately estimated by $\left|\dot{\beta}_{ \pm}\right| \approx \mathcal{M}_{(G)}\left|\beta_{ \pm}\right|$. Since $\mathcal{M}_{(G)}^{2} \propto a^{-6}$ for a small scale factor, the large anisotropic energy at the bouncing point is induced if we consider small bouncing radii. Thus, we conclude that the backreaction on the bouncing universe with large bouncing radii tends to be small.

The backreactions from the other perturbation mode are unclear. However, it is natural to consider that the most symmetric spacetime corresponds to the lowest energy state. Then, one may speculate that the other perturbation modes also lift up the energy for the scale factor like the case of the anisotropic perturbation as (4.34) and (4.35).

We also would like to point out that the oscillating universe obtained by $\mathcal{B}_{\mathcal{O}}^{[1]}$ with $a_{\min } \leq a_{\text {ini }} \leq a_{\max }$ can possibly evolve into a macroscopic universe. In this type of potential $\mathcal{U}(a)$, the oscillating and bouncing solutions are separated by a potential barrier between $a_{\max }$ and $a_{T}$ (see Fig. 1). However, if the potential barrier is sufficiently small, it is possible that the initial oscillating era shifts into an accelerating expanding phase via energy induced by perturbations. In fact, a similar evolution of the universe is found when the spacetime anisotropy is large [15].

\section{CONCLUSION}

To avoid a big-bang singularity at the beginning of the Universe, it is essential to consider the situation in which the null energy condition is violated, i.e., $p+\rho=(1+w) \rho<0$. Based on the HL theory, the higher spatial curvatures in action possibly behave as such exotic matter. Thus, one finds singularity-free cosmological solutions, such as a bouncing universe in non-flat FLRW spacetime. However, it is natural to consider that the effective exotic matter which violates the null energy condition destabilizes spacetime.

In this paper, we investigate the stabilities of bouncing solutions via a perturbation analysis around non-flat FLRW spacetime. Employing (pseudo)spherical harmonic functions, both tensor and scalar perturbations can be decomposed into each $(n ; l, m)$ mode. Then, perturbed actions for tensor and scalar modes at quadratic order are reduced into (3.22) and (3.23), respectively. Note that the integration constant $\mathcal{C}$ induced by the lack of a local Hamiltonian constraint does not affect the quadratic action; however, background dynamics is influenced-i.e., a dustlike additional term is joined in the Friedmann-like equations. In our analysis, the integration constant is set to zero, for simplicity. Thus, the result may be slightly changed if we consider nonzero $\mathcal{C}$, i.e., the spacetime stability around the bouncing point.

In order to avoid ghost instabilities, we must require the coefficients of kinetic terms in the quadratic action to be positive, namely, $\mathcal{F}_{(G)}>0$ and $\mathcal{F}_{(Q)}>0$ for any $a>0$ and perturbation mode $\nu^{2}$. Since $\mathcal{F}_{(G)}=1$, tensor perturbations do not show ghost instability. On the other hand, the condition for ghost avoidance in a scalar perturbation is expressed in terms of $\lambda$ :

$$
\begin{array}{ll}
\lambda \geq 1, & \text { for } K=+1, \\
\lambda>2, & \text { for } K=-1 .
\end{array}
$$


Note that, in flat FLRW spacetime or Minkowski spacetime, the stability condition for a scalar perturbation is given by $\lambda>1$ and the scalar degree of freedom vanishes when $\lambda=1$. It is known that there is no smooth connection between $\lambda>1$ and $\lambda=1$ because of the strong coupling problem. However, in the closed FLRW case, we can take smooth limit $\lambda \rightarrow+1$ without any singular behavior, and then the scalar perturbation can propagate even if the case with $\lambda=1$ is considered. Thus, the limit $\lambda \rightarrow+1$ does not mean GR is restored. The dissimilarity is due to the gauge structure mentioned in Appendix C.

We further consider the positivities of $\mathcal{G}_{(G)}$ and $\mathcal{G}_{(Q)}$ for any perturbation mode $(n ; l, m)$. In order to stabilize the perturbation modes with large $n$, the following conditions must be satisfied:

$$
g_{8} \geq 0, \quad g_{8} \geq 8 g_{7} / 3 .
$$

Although it is possible that $\mathcal{G}_{(G)} \geq 0$ for any $a>0$ and viable $\nu^{2}$, the negativity of $\mathcal{G}_{(Q)}$ cannot be avoided in the infrared regime; i.e., there must be exist $a_{\text {crit }}$ at which any one of the scalar perturbation modes turns from $\mathcal{G}_{(Q)}>0$ to $\mathcal{G}_{(Q)}=0$. This result is consistent with the infrared instability of a scalar graviton in a flat background. Note that the negative value of $\mathcal{G}_{(Q)}$ does not always mean the instability of the scalar perturbation. Then we have investigated the dynamics of perturbations in a bouncing universe via equations of motion.

In the contracting phase, perturbations are possibly amplified by the negative sign of the Hubble term. To suppress the instabilities, the following condition must be satisfied:

$\mathcal{M}_{(G)}^{2} \gtrsim H^{2}, \quad \mathcal{M}_{(Q)}^{2} \gtrsim H^{2} \quad$ for $a_{T}<a \leq a_{\mathrm{ini}}$.

In the infrared regime, the squared effective masses of scalar perturbations must be negative for any choice of the coupling constants $g_{i}$. Thus, we impose the following condition in order to overcome the effect of $\mathcal{M}_{(Q)}^{2}<0$ :

$$
\left|\mathcal{M}_{(Q)}^{2}\right| \lesssim H^{2} \quad \text { for } a \gtrsim a_{\text {crit }}
$$

Thus, the stable bouncing solutions are limited to the case with $\Lambda>0$. If the condition (5.4) is violated, the scalar perturbation is amplified, which means the tachyon instability has occurred. Since $\left|\mathcal{M}_{(Q)}^{2}\right|$ decreases as $a^{-2}$ at the infrared regime, the period for the scalar instability is temporal. The growth rates of the scalar perturbations are related with the minimum value of $\mathcal{M}_{(Q)}^{2} / H^{2}$. Then, small $\lambda-1>0$ and/or large $\Lambda>0$ are preferred to suppress the growth of scalar perturbations. It may be interesting to estimate the permissible growth rate of a scalar perturbation by referring to observational cosmological data. Then, one can derive further constraints to the values of coupling constants.
Additionally, we have investigated the backreaction from a perturbation on the background geometry, especially against an anisotropic perturbation in a $\mathcal{B}_{B C}^{[1]}$-type solution. Considering Bianchi type-IX spacetime with a small anisotropy, the modified Friedmann-like equation including a backreaction from the anisotropic perturbation can be derived as (4.34). It is found that the energy for the scale factor is lifted up by the anisotropic perturbation. Thus, the universe can evolve into a singularity if the potential barrier between $a_{B C}$ and $a_{T}$ is sufficiently small. We also pointed out that the anisotropic energy tends to be large if the bouncing radius is small, because the oscillating amplitudes of $\dot{\beta}_{ \pm}$are enhanced.

We would like to stress that the bouncing solutions in open FLRW spacetime tend to be unstable for the following reasons: (i) The stability conditions for tensor perturbation basically contradict bouncing conditions. Intuitively, $g_{r}>0$ and $g_{s}>0$ are preferred to satisfy $\mathcal{G}_{(G)} \geq 0$ for any $a>0$ and viable $\nu^{2}$. However, we cannot find any types of bouncing solution in open FLRW spacetime under such a condition. Thus, a certain level of tuning of the other coupling constants is required. (ii) After the bounce, to suppress the effect of a negative squared effective mass of the scalar perturbation, strong Hubble friction is significant. However, in open FLRW spacetime, the Hubble friction in such a regime tends to be weak comparing with closed ones, because the coupling constants $\lambda$ must be greater than 2 in order to avoid the ghost instabilities.

Our conclusion is that we have shown that nonsingular cosmological solutions in non-flat FLRW spacetime can be stable against tensor and scalar perturbation, at least at the linear level. Since the projectable HL theory is proved to be truly renormalizable in the perturbation approach, we can calculate the values of the coupling constants via beta functions from the renormalization group, in principle. Thus, it may be possible that the beginning of our Universe can be predicted based on a well-known perturbative quantization approach.

In our case, i.e., the HL theory under a projectability condition, it is indispensable to consider an accelerating expanding phase after the bounce in order to suppress the effect of the negative squared effective mass of the scalar perturbation in the infrared regime. We speculate that the scalar instabilities in the infrared regime are the nature of the projectable HL gravity theory. This infrared pathological behavior is conceivably resolved by considering the extended theory, i.e., nonprojectable HL gravity whose scalar graviton can be stable at least in Minkowski spacetime.

We additionally mention that the infrared limit of the nonprojectable HL theory (i.e., without higher spatial curvatures) is included within the framework of the Horndeski theory, which is the general theory of ghost-free scalar-tensor gravity [30]. Based on the Horndeski theory, it turns out that any nonsingular cosmological solution is 
unstable [31]. On the other hand, it is worth mentioning that the no-go theorem for the stable nonsingular solution can be violated if the extended theory including higher spatial curvatures is considered [32]. Thus, in view of the situation, it should be interesting to investigate the stability of bouncing solutions with higher spatial curvatures based on the nonprojectable HL theory as a special case of extended Horndeski scalar-tensor gravity [33].

\section{ACKNOWLEDGMENTS}

The authors thank Kei-ichi Maeda, Shuntaro Mizuno, and Katsuki Aoki for valuable comment and discussions.

\section{APPENDIX A: SPHERICAL AND PSEUDOSPHERICAL HARMONICS}

The detailed discussion about the tensor (pseudo)spherical harmonics has already been performed in Refs. [26,27]. In this section, we give the correspondence between our definition and those of the above references.

\section{Tensor spherical harmonics on a two-sphere}

Before considering the three-dimensional case, we introduce spherical harmonics on a unit two-sphere whose metric $s_{A B}$ is given by

$$
d s_{(2)}^{2}=d \theta^{2}+\sin ^{2} \theta d \phi^{2} .
$$

Then, the scalar spherical harmonics $Y^{(l m)}(\theta, \phi)$ is given by

$$
\begin{aligned}
Y^{(l m)}= & (-1)^{(m+|m|) / 2} \sqrt{\frac{(2 l+1)(l-|m|) !}{4 \pi(l+|m|) !}} \\
& \times P_{l}^{|m|}(\cos \theta) e^{i m \phi},
\end{aligned}
$$

where $P_{l}^{|m|}(\cos \theta)$ is a Legendre polynomial whose Rodrigues formula is given by

$$
P_{n}^{m}(x)=\frac{(-1)^{n}}{2^{n} n !}\left(1-x^{2}\right)^{m / 2} \frac{d^{n+m}}{d x^{n+m}}\left(1-x^{2}\right)^{n},
$$

where degrees $l, m \in \mathbb{Z}$ are constrained by $0 \leq|m| \leq l$.

The vector spherical harmonic functions are classified into two classes. Since the vector quantity can be decomposed into a gradient part and a rotational part, we define the gradient of the scalar harmonics $\psi_{A}^{(l m)}$ and the dual of the gradient $\phi_{A}^{(l m)}$ :

$$
\begin{gathered}
\psi_{A}^{(l m)}:=\mathcal{D}_{A} Y^{(l m)}, \\
\phi_{A}^{(l m)}:=\epsilon_{A}{ }^{B} \mathcal{D}_{B} Y^{(l m)},
\end{gathered}
$$

where $\mathcal{D}_{A}$ denotes a covariant derivative on the two-sphere and $\epsilon_{A B}$ is the Levi-Civita tensor on the two-sphere. Note that $\psi_{A}$ and $\phi_{A}$ possess even and odd parity, respectively.

The tensoral ones are classified into three types: $\eta_{A B}$ is a trace part, which is proportional to a two-metric. $\psi_{A B}$ and $\phi_{A B}$ are traceless with even and odd parity, respectively. The explicit forms are given by

$$
\eta_{A B}^{(l m)}:=Y^{(l m)} s_{A B},
$$

$$
\begin{gathered}
\psi_{A B}^{(l m)}:=\mathcal{D}_{A} \mathcal{D}_{B} Y^{(l m)}+\frac{l(l+1)}{2} Y^{(l m)} s_{A B}, \\
\phi_{A B}^{(l m)}=\frac{1}{2}\left[\mathcal{D}_{A} \phi_{B}^{(l m)}+\mathcal{D}_{B} \phi_{A}^{(l m)}\right] .
\end{gathered}
$$

\section{Tensor harmonics on a three-(pseudo)sphere}

We consider the harmonics on a unit three-sphere and a unit three-pseudosphere whose metrics $\hat{\gamma}_{i j}$ and $\check{\gamma}_{i j}$ are given, respectively, by

$$
\begin{aligned}
d \hat{\ell}^{2} & =d \chi^{2}+\sin ^{2} \chi\left(d \theta^{2}+\sin ^{2} \theta d \phi^{2}\right), \\
d \check{\ell}^{2} & =d \chi^{2}+\sinh ^{2} \chi\left(d \theta^{2}+\sin ^{2} \theta d \phi^{2}\right) .
\end{aligned}
$$

To construct the harmonics for each cases, we require that (i) the harmonic function $\mathbf{Y}$ has the eigenvalues of the Laplace-Beltrami operator and (ii) the orthonormality is satisfied. Then, the functions defined on the three-sphere and three-pseudosphere can be expanded by each $(n, l, m)$ mode of harmonics. For the spherical case, $n \geq 1$ is a discrete natural number which constrains $0 \leq l \leq n-1$. On the other hand, for the pseudospherical case, $n \geq 1$ is defined as a continuous number.

We give the explicit form of the scalar spherical harmonics $\hat{Y}^{(n ; l m)}$ :

$$
\begin{aligned}
\hat{Y}^{(n ; l m)}(\chi, \theta, \phi) & :=\hat{X}^{(n ; l)}(\chi) Y^{(l m)}(\theta, \phi), \\
\hat{X}^{(n ; l)}(\chi):= & \sqrt{\frac{2}{\pi}}\left[\prod_{0 \leq k \leq l} \frac{1}{n^{2}-k^{2}}\right]^{1 / 2} \\
& \times \sin ^{l} \chi \frac{d^{(l+1)}}{d(\cos \chi)^{l+1}} \cos (n \chi) .
\end{aligned}
$$

Note that $\hat{X}^{(n ; l)}$ is expressed in terms of Gegenbauer (ultraspherical) polynomials which is a generalization of Legendre polynomials [26,34]. The eigenvalues of the Laplace-Beltrami operator are given by

$$
\hat{\mathcal{D}}^{2} \hat{Y}^{(n ; l m)}=-\left(n^{2}-1\right) \hat{Y}^{(n ; l m)},
$$

where $\hat{\mathcal{D}}_{i}$ denotes a covariant derivative in terms of $\hat{\gamma}_{i j}$. One can confirm that the above harmonics satisfy orthonormality: 


$$
\left\langle\hat{Y}^{(n ; l m)}, \hat{Y}^{\left(n^{\prime} ; l^{\prime} m^{\prime}\right)}\right\rangle=\delta_{\left(n, n^{\prime}\right)} \delta_{\left(l, l^{\prime}\right)} \delta_{\left(m, m^{\prime}\right)},
$$

where we define the internal product on the threesphere as

$$
\left\langle\hat{\mathbf{Y}}_{1}, \hat{\mathbf{Y}}_{2}\right\rangle:=\int_{0}^{\pi} d \chi \int_{0}^{\pi} d \theta \int_{0}^{2 \pi} d \phi \sqrt{\hat{\gamma}} \hat{\mathbf{Y}}_{1} \cdot \hat{\mathbf{Y}}_{2}
$$

and a symbol - denotes a contraction of tensor indices.

The pseudospherical harmonics $\check{Y}^{(n ; l m)}$ can be derived from the three-spherical ones by considering an analytic continuation, namely, $\chi \rightarrow i \chi$ and $n \rightarrow$ in [25,27]:

$$
\begin{aligned}
\check{Y}^{(n ; l m)} & =\check{X}^{(n ; l)}(\chi) Y^{(l m)}(\theta, \phi), \\
\check{X}^{(n ; l)}= & \sqrt{\frac{2}{\pi}}\left[\prod_{0 \leq k \leq l} \frac{1}{n^{2}+k^{2}}\right]^{1 / 2} \\
& \times \sinh ^{l} \chi \frac{d^{(l+1)}}{d(\cosh \chi)^{l+1}} \cos (n \chi) .
\end{aligned}
$$

The eigenvalues of the Laplace-Beltrami operator on a unit three-pseudosphere are given by

$$
\check{\mathcal{D}}^{2} \breve{Y}^{(n ; l m)}=-\left(n^{2}+1\right) \check{Y}^{(n ; l m)},
$$

where $\check{\mathcal{D}}_{i}$ denotes a covariant derivative in terms of $\check{\gamma}_{i j}$. The orthonormality is also satisfied if we define the internal product on the three unit pseudosphere as follows:

$$
\left\langle\check{\mathbf{Y}}_{1}, \check{\mathbf{Y}}_{2}\right\rangle:=\lim _{L \rightarrow \infty} \frac{\pi}{L} \int_{0}^{L} d \chi \int_{0}^{\pi} d \theta \int_{0}^{2 \pi} d \phi \sqrt{\check{\gamma}} \check{\mathbf{Y}}_{1} \cdot \check{\mathbf{Y}}_{2}
$$

Then, the orthonormality of pseudospherical harmonics is given by

$$
\left\langle\check{Y}^{(n ; l m)}, \check{Y}^{\left(n^{\prime} ; l^{\prime} m^{\prime}\right)}\right\rangle=\delta\left(n-n^{\prime}\right) \delta_{\left(l, l^{\prime}\right)} \delta_{\left(m, m^{\prime}\right)} .
$$

In order to unify the discussion of both cases, we define the eigenvalues of spherical and pseudospherical harmonics as follows:

$$
\nu^{2}:=\left\{\begin{array}{lll}
n^{2}-1, & n \in \mathbb{N} & \text { for } K=1, \\
n^{2}+1, & n \in \mathbb{R} & \text { for } K=-1 .
\end{array}\right.
$$

In what follows, we abbreviate the superscripts ${ }^{\wedge}$ and ${ }^{\wedge}$ if not otherwise specified.

\section{a. Scalar type}

We introduce the scalar-type harmonics which contribute to the scalar perturbation. Since the scalar quantities have already been introduced in the previous part, we focus only on the vector and tensor quantities.
The vector quantities $C_{i}$ are defined by

$$
C_{i}^{(n ; l m)}=\mathcal{D}_{i} Y^{(n ; l m)} .
$$

The tensor quantities are classified into two kinds:

$$
\begin{gathered}
C_{i j}^{(n ; l m)}=\mathcal{D}_{i} \mathcal{D}_{j} Y^{(n ; l m)}+\frac{\nu^{2}}{3} Y^{(n ; l m)} \gamma_{i j}, \\
D_{i j}^{(n ; l m)}=Y^{(n ; l m)} \gamma_{i j} .
\end{gathered}
$$

Namely, $C_{i j}$ and $D_{i j}$ assume the traceless and trace parts, respectively. Considering the internal products, the normalized scalar-type harmonics are defined by

$$
\begin{gathered}
Q^{(n ; l m)}:=Y^{(n ; l m)}, \\
Q_{i}^{(n ; l m)}:=\nu^{-1} C_{i}^{(n ; l m)}, \\
Q_{i j}^{(n ; l m)}:=\frac{1}{\sqrt{3}} D_{i j}^{(n ; l m)}, \\
P_{i j}^{(n ; l m)}:=\left[\frac{2}{3} \nu^{2}\left(\nu^{2}-3 K\right)\right]^{-1 / 2} C_{i j}^{(n ; l m)} .
\end{gathered}
$$

When we consider the spherical (pseudospherical) case, the spatial curvature takes $K=1(K=-1)$.

\section{b. Vector type}

The vector-type harmonics contribute to the transverse modes of the metric perturbation. Namely, the divergences of these harmonics vanish.

The vector quantities include two types of harmonics. One is odd parity mode $A_{i}$, whose explicit form is given by

$$
A_{i}^{(n ; l m)}=\left(0, f(\chi) X^{(n ; l)} \phi_{A}^{(l m)}\right),
$$

where the function $f(\chi)$ is defined in (2.7). Then, the eigenvalues are given by

$$
\mathcal{D}^{2} A_{i}^{(n ; l m)}=-\left(\nu^{2}-K\right) A_{i}^{(n ; l m)} .
$$

The other is even parity mode $B_{i}$, whose explicit form is given by

$$
B_{i}^{(n ; l m)}=-\epsilon_{i}{ }^{j k} \mathcal{D}_{j} A_{k}^{(n ; l m)},
$$

where $\epsilon_{i j k}$ is the Levi-Civita symbol associated with $\gamma_{i j}$. The eigenvalues are as the same as the odd ones.

The tensor quantities can be constructed by taking a symmetrized gradient of each vector quantity:

$$
A_{i j}^{(n ; l m)}=\frac{1}{2}\left[\mathcal{D}_{i} A_{j}^{(n ; l m)}+\mathcal{D}_{j} A_{i}^{(n ; l m)}\right],
$$


TABLE IV. The properties of spherical and pseudospherical harmonics. $K= \pm 1$ denotes the spatial curvature. The circle represents that the corresponding calculation can produce a nonzero value. On the other hand, the cross denotes that the calculation always gives zero. N/A means that the corresponding calculation is prohibited.

\begin{tabular}{|c|c|c|c|c|c|c|}
\hline Type & $\mathbf{Y}$ & Parity & Trace & Divergence & Eigenvalues & Viable degrees \\
\hline Scalar & $Q^{(n ; l m)}$ & Even & N/A & N/A & $-\nu^{2}$ & $n \geq 1$ \\
\hline Scalar & $Q_{i}^{(n ; l m)}$ & Even & N/A & 0 & $-\nu^{2}+2 K$ & $\begin{array}{ll}n \geq 2 & \text { for } K=1 \\
n \geq 1 & \text { for } K=-1\end{array}$ \\
\hline Scalar & $Q_{i j}^{(n ; l m)}$ & Even & $\bigcirc$ & $\bigcirc$ & $-\nu^{2}$ & $n \geq 1$ \\
\hline Scalar & $P_{i j}^{(n ; l m)}$ & Even & $x$ & $\bigcirc$ & $-\nu^{2}+6 K$ & $\begin{array}{ll}n \geq 3 & \text { for } K=1 \\
n \geq 1 & \text { for } K=-1\end{array}$ \\
\hline Vector & $S_{i}^{(n ; l m)}$ & Odd and even & N/A & $x$ & $-\nu^{2}+K$ & $l \geq 1$ \\
\hline Vector & $S_{i j}^{(n ; l m)}$ & Odd and even & $x$ & $\bigcirc$ & $-\nu^{2}+5 K$ & $l \geq 1$ \\
\hline Tensor & $G_{i j}^{(n ; l m)}$ & Odd and even & $x$ & $x$ & $-\nu^{2}+2 K$ & $l \geq 2$ \\
\hline
\end{tabular}

$$
B_{i j}^{(n ; l m)}=\frac{1}{2}\left[\mathcal{D}_{i} B_{j}^{(n ; l m)}+\mathcal{D}_{j} B_{i}^{(n ; l m)}\right] .
$$

The eigenvalues are given by

$$
\mathcal{D}^{2} A_{i j}^{(n ; l m)}=-\left(\nu^{2}-5 K\right) A_{i j}^{(n ; l m)}
$$

The even parity modes have the identical eigenvalue as the odd ones. Then, the normalized vector-type harmonics are defined by

$$
\begin{gathered}
S_{(o) i}^{(n ; l m)}:=[l(l+1)]^{-1 / 2} A_{i}^{(n ; l m)}, \\
S_{(e) i}^{(n ; l m)}:=\left[l(l+1)\left(\nu^{2}+K\right)\right]^{-1 / 2} B_{i}^{(n ; l m)}, \\
S_{(o) i j}^{(n ; l m)}:=\left[\frac{l(l+1)}{2}\left(\nu^{2}-3 K\right)\right]^{-1 / 2} A_{i j}^{(n ; l m)}, \\
S_{(e) i j}^{(n ; l m)}:=\left[\frac{l(l+1)}{2}\left(\nu^{2}-3 K\right)\left(\nu^{2}+K\right)\right]^{-1 / 2} B_{i j}^{(n ; l m)} .
\end{gathered}
$$

\section{c. Tensor type}

The tensor-type harmonics contribute to the transversetraceless mode of perturbation. Thus, we find only tensor quantities in this type. As is the case with the vector-type harmonics, there are odd and even parity modes. The odd parity modes $E_{i j}^{(n ; l m)}$ are given by

$$
\begin{gathered}
E_{\chi \chi}^{(n ; l m)}=0, \\
E_{\chi A}^{(n ; l m)}=X^{(n ; l)} \phi_{A}^{(l m)}, \\
E_{\chi A}^{(n ; l m)}=\left[\frac{2}{(l+2)(l-1)}\right] \frac{d}{d \chi}\left[f(\chi)^{2} X^{(n ; l)}\right] \phi_{A B}^{(l m)} .
\end{gathered}
$$

The eigenvalue equation is given by

$$
\mathcal{D}^{2} E_{i j}^{(n ; l m)}=-\left(\nu^{2}-2 K\right) E_{i j}^{(n ; l m)}
$$

Those of even parity $F_{i j}^{(n ; l m)}$ are given by

$$
F_{i j}^{(n ; l m)}=\frac{1}{2}\left[\epsilon_{i}^{p q} \mathcal{D}_{p} E_{q j}^{(n ; l m)}+\epsilon_{j}^{p q} \mathcal{D}_{p} E_{q i}^{(n ; l m)}\right] .
$$

The eigenvalues are as the same as the odd ones. Then, the normalized tensor-type harmonics are defined by

$$
\begin{gathered}
G_{(o) i j}^{(n ; l m)}:=\left[\frac{2 l(l+1)}{(l+2)(l-1)} \nu^{2}\right]^{-1 / 2} E_{i j}^{(n ; l m)}, \\
G_{(e) i j}^{(n ; l m)}:=\left[\frac{2 l(l+1)}{(l+2)(l-1)} \nu^{2}\left(\nu^{2}+K\right)\right]^{-1 / 2} F_{i j}^{(n ; l m)} .
\end{gathered}
$$

We shall summarize the properties of the spherical and pseudospherical harmonics in Table IV.

\section{APPENDIX B: THE FORMULAS OF HARMONICS IN NONFLAT FLRW SPACE}

In this section, the traces, covariant derivatives, and norms of tensor harmonics in nonflat FLRW space are shown. Since both the odd and even parity modes share the properties, we abbreviate the subscript of parity. Note that the results in flat FLRW spacetime are formally reproduced if we take $K=0$ in the following expressions.

\section{Gradients}

The gradients of the normalized scalar harmonics are given by

$$
\nabla_{i} Q^{(n ; l m)}=\nu Q_{i}^{(n ; l m)},
$$

where $\nabla_{i}$ is a covariant derivative associated with the induced metric of nonflat FLRW space $g_{i j}$. The symmetrized gradients of the normalized vector harmonics are given by 


$$
\begin{gathered}
\nabla_{(i} Q_{j)}^{(n ; l m)}=-\frac{\nu}{\sqrt{3}} Q_{i j}^{(n ; l m)}+\sqrt{\frac{2}{3}\left(\nu^{2}-3 K\right)} P_{i j}^{(n ; l m)}, \\
\nabla_{(i} S_{j)}^{(n ; l m)}=\sqrt{\frac{\nu^{2}-3 K}{2}} S_{i j}^{(n ; l m)} .
\end{gathered}
$$

\section{Traces}

The traces of the normalized harmonics are given by

$$
g^{i j} Q_{i j}^{(n ; l m)}=\frac{\sqrt{3}}{a^{2}} Q^{(n ; l m)},
$$

and the others all vanish.

\section{Divergences}

The divergences of the normalized vector harmonics vanish except $Q_{i}^{(n ; l m)}$ :

$$
g^{i j} \nabla_{i} Q_{j}^{(n ; l m)}=-\frac{\nu}{a^{2}} Q^{(n ; l m)} .
$$

The nontrivial divergences of the normalized spherical harmonics are given by

$$
\begin{gathered}
g^{j k} \nabla_{k} Q_{i j}^{(n ; l m)}=\frac{\nu}{\sqrt{3} a^{2}} Q_{i}^{(n ; l m)}, \\
g^{j k} \nabla_{k} P_{i j}^{(n ; l m)}=-\frac{1}{a^{2}} \sqrt{\frac{2\left(\nu^{2}-3 K\right)}{3}} Q_{i}^{(n ; l m)}, \\
g^{j k} \nabla_{k} S_{i j}^{(n ; l m)}=-\frac{1}{a^{2}} \sqrt{\frac{\nu^{2}-3 K}{2}} S_{i}^{(n ; l m)} .
\end{gathered}
$$

\section{Laplace-Beltrami operator}

The eigenvalues of the Laplace-Beltrami operator in nonflat FLRW space are listed. Those of scalar-type harmonics are given by

$$
\begin{gathered}
\nabla^{2} Q^{(n ; l m)}=-\frac{\nu^{2}}{a^{2}} Q^{(n ; l m)}, \\
\nabla^{2} Q_{i}^{(n ; l m)}=-\left(\frac{\nu^{2}-2 K}{a^{2}}\right) Q_{i}^{(n ; l m)}, \\
\nabla^{2} Q_{i j}^{(n ; l m)}=-\frac{\nu^{2}}{a^{2}} Q_{i j}^{(n ; l m)}, \\
\nabla^{2} P_{i j}^{(n ; l m)}=-\left(\frac{\nu^{2}-6 K}{a^{2}}\right) P_{i j}^{(n ; l m)},
\end{gathered}
$$

those of vector-type are given by

$$
\begin{aligned}
& \nabla^{2} S_{i}^{(n ; l m)}=-\left(\frac{\nu^{2}-K}{a^{2}}\right) S_{i}^{(n ; l m)}, \\
& \nabla^{2} S_{i j}^{(n ; l m)}=-\left(\frac{\nu^{2}-5 K}{a^{2}}\right) S_{i j}^{(n ; l m)},
\end{aligned}
$$

and those of tensor-type are given by

$$
\nabla^{2} G_{i j}^{(n ; l m)}=-\left(\frac{\nu^{2}-2 K}{a^{2}}\right) G_{i j}^{(n ; l m)} .
$$

\section{APPENDIX C: GAUGE FIXING}

The metric perturbations include both physical and gauge degrees of freedom. Thus, we can perform a further simplification by fixing the gauge. Note that the HL theory loses the general covariance because of Lifshitz scaling; i.e., the rotational transformation of the time direction is prohibited. Thus, the infinitesimal coordinate transformation is expressed as follows:

$$
t \rightarrow t+f(t), \quad x^{i} \rightarrow x^{i}+\zeta^{i}\left(t, x^{j}\right) .
$$

Consider the FLRW background:

$$
\bar{N}=1, \quad \bar{N}_{i}=0, \quad \bar{g}_{i j}=a^{2} \gamma_{i j},
$$

where $\gamma_{i j}$ corresponds to the three Euclid space $(K=0)$, unit sphere $(K=1)$, or unit pseudosphere $(K=-1)$. Then, the infinitesimal transformations of the perturbed ADM quantities are given by

$$
\begin{gathered}
\alpha^{(\text {gauge })}=-\partial_{t} f, \\
\beta_{i}^{\text {(gauge })}=\partial_{t} \zeta_{i}-2 H \zeta_{i}, \\
h_{i j}^{\text {(gauge })}=2 \nabla_{(i} \zeta_{j)}-2 H f \bar{g}_{i j} .
\end{gathered}
$$

We define the harmonic expansion of $\zeta^{i}$ as follows:

$$
\begin{aligned}
\zeta_{i}= & \sum_{n, l, m} a^{2}\left[\zeta_{(Q)}^{(n ; l m)} Q_{i}^{(n ; l m)}\right. \\
& \left.+\zeta_{(S ; o)}^{(n ; l m)} S_{(o) i}^{(n ; l m)}+\zeta_{(S ; e)}^{(n ; l m)} S_{(e) i}^{(n ; l m)}\right] .
\end{aligned}
$$

Since $f$ does depend only on the time, we do not have to expand by the harmonics. Then, we can explicitly describe the gauge transformations of perturbed ADM variables.

We first consider the transformation of the scalar perturbation:

$$
\begin{gathered}
\alpha \rightarrow \alpha-\partial_{t} f, \\
\beta_{(Q)}^{(n ; l m)} \rightarrow \beta_{(Q)}^{(n ; l m)}+\left[\partial_{t}-2 H\right] \zeta_{(Q)}^{(n ; l m)},
\end{gathered}
$$




$$
h_{(P)}^{(n ; l m)} \rightarrow h_{(P)}^{(n ; l m)}+\frac{2}{a^{2}} \sqrt{\frac{2\left(\nu^{2}-3 K\right)}{3}} \zeta_{(Q)}^{(n ; l m)},
$$

and

$$
\begin{aligned}
& \sum_{n, l, m} h_{(Q)}^{(n ; l m)} Q_{i j}^{(n ; l m)} \\
& \quad \rightarrow \sum_{n, l, m}\left[h_{(Q)}^{(n ; l m)}-\frac{2 \nu}{\sqrt{3} a^{2}} \zeta_{(Q)}^{(n ; l m)}\right] Q_{i j}^{(n ; l m)}-2 \sqrt{3} H f \bar{g}_{i j} .
\end{aligned}
$$

Those of the vector perturbation are given by

$$
\begin{gathered}
\beta_{(S)}^{(n ; l m)} \rightarrow \beta_{(S)}^{(n ; l m)}+\left[\partial_{t}-2 H\right] \zeta_{(S)}^{(n: l m)}, \\
h_{(S)}^{(n ; l m)} \rightarrow h_{(S)}^{(n ; l m)}+\sqrt{2\left(\nu^{2}-3 K\right)} \zeta_{(S)}^{(n ; l m)} .
\end{gathered}
$$

Since both odd and even parity modes obey the same transformation law, the parity subscripts are abbreviated. Those of the tensor perturbation are given by

$$
h_{(G)}^{(n ; l m)} \rightarrow h_{(G)}^{(n ; l m)}
$$

where the parity subscripts are also abbreviated for the same reason as the vector perturbation. Note that the tensor perturbation is gauge invariant.
Then, we consider the gauge fixing to simplify the procedure for perturbation. In the scalar perturbation, we find two types of quantities which can be manipulated, i.e., $f$ and $\zeta_{(Q)}$. Obviously, $h_{(P)}$, which is the traceless part of the scalar perturbation, can be eliminated by choosing

$$
\zeta_{(Q)}^{(n ; l m)}=-\frac{a^{2}}{2}\left[\frac{2\left(\nu^{2}-3 K\right)}{3}\right]^{-1 / 2} h_{(P)}^{(n ; l m)} .
$$

However, the trace part $h_{(Q)}$ is not, because the transformation law (C10) includes a spatially homogeneous part which is proportional to $f \bar{g}_{i j}$. Since the terms which include $\dot{h}_{(Q)}$ join the quadratic action, the scalar degree of freedom appears in this theory unlike the case of GR. Instead, we eliminate the lapse perturbation $\alpha$ by solving differential equation $\partial_{t} f=\alpha$.

In the vector perturbation, $\zeta_{(S ; o)}$ and $\zeta_{(S ; e)}$ can be manipulated; thus, we eliminate $h_{(S ; o)}$ and $h_{(S ; e)}$ by choosing

$$
\begin{aligned}
& \zeta_{(S ; o)}^{(n ; l m)}=-\left[2\left(\nu^{2}-3 K\right)\right]^{-1 / 2} h_{(S ; o)}^{(n ; l m)}, \\
& \zeta_{(S ; e)}^{(n ; l m)}=-\left[2\left(\nu^{2}-3 K\right)\right]^{-1 / 2} h_{(S ; e)}^{(n ; l m)} .
\end{aligned}
$$

To recap, we can eliminate the following perturbation modes by choosing the gauge:

$$
\alpha=h_{(P)}^{(n ; l m)}=h_{(S ; o)}^{(n ; l m)}=h_{(S ; e)}^{(n ; l m)}=0 .
$$

[1] R. Penrose, Gravitational Collapse and Space-Time Singularities, Phys. Rev. Lett. 14, 57 (1965); S. W. Hawking, The Occurrence of Singularities in Cosmology. III. Causality and Singularities, Proc. R. Soc. A 300, 187 (1967); S. W. Hawking and R. PenroseThe Singularities of Gravitational Collapse and Cosmology, Proc. R. Soc. A 314, 529 (1970); S. W. Hawking and G. F. R. Ellis, The Large Scale Structure of Space-Time (Cambridge University Press, Cambridge, England, 1973).

[2] A. Borde, A. H. Guth, and A. Vilenkin, Inflationary Spacetimes Are Not Past-Complete, Phys. Rev. Lett. 90, 151301 (2003).

[3] See, for example, M. Novello and S. E. P. Bergliaffa, Bouncing cosmologies, Phys. Rep. 463, 127 (2008), and references therein.

[4] M. B. Green, J. H. Schwarz, and E. Witten, Superstring Theory (Cambridge University Press, Cambridge, England, 1987); J. Polchinski, String Theory (Cambridge University Press, Cambridge, England, 1998).

[5] See, for example, C. Rovelli, Quantum Gravity (Cambridge University Press, Cambridge, England, 2004).

[6] R. Loll, Discrete Lorentzian quantum gravity, Nucl. Phys. B, Proc. Suppl. 94, 96 (2001).
[7] T. Biswas, E. Gerwick, T. Koivisto, and A. Mazumdar, Towards Singularity and Ghost-Free Theories of Gravity, Phys. Rev. Lett. 108, 031101 (2012); T. Biswas, A. S. Koshelev, A. Mazumdar, and S. Y. Vernov, Stable bounce and inflation in non-local higher derivative cosmology, J. Cosmol. Astropart. Phys. 08 (2012) 024.

[8] P. Hořava, Quantum gravity at a Lifshitz point, Phys. Rev. D 79, 084008 (2009).

[9] E. M. Lifshitz, On the theory of second-order phase transitions I \& II, Zh. Eksp. Teor. Fiz. 11, 255 (1941).

[10] A. O. Barvinsky, D. Blas, M. Herrero-Valea, S. M. Sibiryakov, and C.F. Steinwachs, Renormalization of Horava gravity, Phys. Rev. D 93, 064022 (2016).

[11] D. Blas and S. Sibiryakov, Hořava gravity versus thermodynamics: The black hole case, Phys. Rev. D 84, 124043 (2011); E. Barausse, T. Jacobson, and T. P. Sotiriou, Black holes in Einstein-aether and Hořava-Lifshitz gravity, Phys. Rev. D 83, 124043 (2011); P. Berglund, J. Bhattacharyya, and D. Mattingly, Mechanics of universal horizons, Phys. Rev. D 85, 124019 (2012); Y. Misonoh and K. Maeda, Black holes and thunderbolt singularities with Lifshitz scaling terms, Phys. Rev. D 92, 084049 (2015). 
[12] E. Kiritsis and G. Kofinas, Horava-Lifshitz cosmology, Nucl. Phys. B821, 467 (2009); R. H. Brandenberger, Matter bounce in Horava-Lifshitz cosmology, Phys. Rev. D 80, 043516 (2009).

[13] T. Ha, Y. Huang, Q. Ma, K. D. Pechan, T. J. Renner, Z. Wu, G. A. Benesh, and A. Wang, Classification of the FRW universe with a cosmological constant and a perfect fluid of the equation of state $p=w \rho$, Gen. Relativ. Gravit. 44, 1433 (2012); A. Wang and Y. Wu, Thermodynamics and classification of cosmological models in the Horava-Lifshitz theory of gravity, J. Cosmol. Astropart. Phys. 07 (2009) 012.

[14] K. Maeda, Y. Misonoh, and T. Kobayashi, Oscillating universe in Horava-Lifshitz gravity, Phys. Rev. D 82, 064024 (2010).

[15] Y. Misonoh, K. Maeda, and T. Kobayashi, Oscillating Bianchi IX universe in Horava-Lifshitz gravity, Phys. Rev. D 84, 064030 (2011).

[16] R. Cai, B. Hu, and H. Zhang, Dynamical scalar degree of freedom in Horava-Lifshitz gravity, Phys. Rev. D 80, 041501 (2009); K. Yamamoto, T. Kobayashi, and G. Nakamura, Breaking the scale invariance of the primordial power spectrum in Horava-Lifshitz cosmology, Phys. Rev. D 80, 063514 (2009); Y. Lu and Y. Piao, Scale invariance from modified dispersion relations, Int. J. Mod. Phys. D 19, 1905 (2010); T. Kobayashi, Y. Urakawa, and M. Yamaguchi, Large scale evolution of the curvature perturbation in Horava-Lifshitz cosmology, J. Cosmol. Astropart. Phys. 11 (2009) 015; Cosmological perturbations in a healthy extension of Horava gravity, J. Cosmol. Astropart. Phys. 04 (2010) 025; K. Izumi, T. Kobayashi, and S. Mukohyama, Non-Gaussianity from Lifshitz scalar, J. Cosmol. Astropart. Phys. 10 (2010) 031.

[17] C. Charmousis, G. Niz, A. Padilla, and P. M. Saffin, Strong coupling in Horava gravity, J. High Energy Phys. 08 (2009) 070; M. Li and Y. Pang, A trouble with Horava-Lifshitz gravity, J. High Energy Phys. 08 (2009) 015; D. Blas, O. Pujolas, and S. Sibiryakov, On the extra mode and inconsistency of Horava gravity, J. High Energy Phys. 10 (2009) 029; C. Bogdanos and E. N. Saridakis, Perturbative instabilities in Horava gravity, Classical Quantum Gravity 27, 075005 (2010); S. Mukohyama, Horava-Lifshitz cosmology: A review, Classical Quantum Gravity 27, 223101 (2010).

[18] D. Blas, O. Pujolas, and S. Sibiryakov, Consistent Extension of Hořava Gravity, Phys. Rev. Lett. 104, 181302 (2010).

[19] Y. Q. Huang, A. Wang, and Q. Wu, Stability of the de Sitter spacetime in Horava-Lifshitz theory, Mod. Phys. Lett. A 25, 2267 (2010); A. Wang and Q. Wu, Stability of spin-0 graviton and strong coupling in Horava-Lifshitz theory of gravity, Phys. Rev. D 83, 044025 (2011).

[20] A. Wang and R. Maartens, Cosmological perturbations in Horava-Lifshitz theory without detailed balance, Phys. Rev. D 81, 024009 (2010).
[21] X. Gao, Y. Wang, W. Xue, and R. H. Brandenberger, Fluctuations in a Horava-Lifshitz bouncing cosmology, J. Cosmol. Astropart. Phys. 02 (2010) 020.

[22] T. P. Sotiriou, M. Visser, and S. Weinfurtner, Phenomenologically Viable Lorentz-Violating Quantum Gravity, Phys. Rev. Lett. 102, 251601 (2009); Quantum gravity without Lorentz invariance, J. High Energy Phys. 10 (2009) 033.

[23] S. M. Carrollv and E. A. Lim, Lorentz-violating vector fields slow the Universe down, Phys. Rev. D 70, 123525 (2004); B. Z. Foster and T. Jacobson, Post-Newtonian parameters and constraints on Einstein-aether theory, Phys. Rev. D 73, 064015 (2006).

[24] S. Mukohyama, Dark matter as integration constant in Horava-Lifshitz gravity, Phys. Rev. D 80, 064005 (2009).

[25] E. M. Lifshitz and I. M. Khalatnikov, Investigations in relativistic cosmology, Adv. Phys. 12, 185 (1963).

[26] V. D. Sandberg, Tensor spherical harmonics on $S^{2}$ and $S^{3}$ as eigenvalues problems, J. Math. Phys. (N.Y.) 19, 2441 (1978).

[27] K. Tomita, Tensor spherical and pseudo-spherical harmonics in four-dimensional spaces, Prog. Theor. Phys. 68, 310 (1982).

[28] R. M. Wald, Asymptotic behavior of homogeneous cosmological models in the presence of a positive cosmological constant, Phys. Rev. D 28, 2118 (1983); I. Moss and V. Sahni, Anisotropy in the chaotic inflationary universe, Phys. Lett. B 178, 159 (1986); J. D. Barrow, Cosmic nohair theorem and inflation, Phys. Lett. B 187, 12 (1987); L. G. Jensen and J. A. Stein-Schabes, Is inflation natural?, Phys. Rev. D 35, 1146 (1987).

[29] See, for example, M. P. Ryan and L. C. Shepley, Homogeneous Relativistic Cosmologies (Princeton University, Princeton, NJ, 1975); F. Sato and H. Kodama, General Relativity (Iwanami, Tokyo, 1992).

[30] G. W. Horndeski, Second-order scalar-tensor field equations in a four-dimensional space, Int. J. Theor. Phys. 10, 363 (1974); T. Kobayashi, M. Yamaguchi, and J. Yokoyama, Generalized G-inflation: Inflation with the most general second-order field equations, Prog. Theor. Phys. 126, 511 (2011).

[31] T. Kobayashi, Generic instabilities of non-singular cosmologies in Horndeski theory: A no-go theorem, Phys. Rev. D 94, 043511 (2016); A. Ijjas and P. J. Steinhardt, Classically Stable Nonsingular Cosmological Bounces, Phys. Rev. Lett. 117, 121304 (2016).

[32] Y. Cai, Y. Wan, H-G. Li, T. Qiu, and Y-S. Piao, The effective field theory of nonsingular cosmology, J. High Energy Phys. 01 (2017) 090.

[33] Y. Misonoh, M. Fukushima, and S. Miyashita (to be published).

[34] M. Abramowitz and I. A. Stegun, Handbook of Mathematical Functions with Formulas, Graphs, and Mathematical Tables (Dover, New York, 1965). 